\title{
The plastic flow stability of chip materials in metal cutting process
}

\author{
Wei Ma ${ }^{1} \cdot$ Fei Shuang ${ }^{1,2}$
}

Received: 10 October 2018 / Accepted: 23 August 2019/Published online: 23 August 2019

(C) Springer-Verlag London Ltd., part of Springer Nature 2019

\begin{abstract}
This study investigates the chip formation mechanism and relevant plastic flow stability in the orthogonal cutting process (OCP) through high-speed cutting experiments and theoretical modelling for four types of metals. The chip morphology transitions from continuous to serrated and to continuous again are observed with critical speeds depending on the work material properties and cutting conditions. To exam the influence of two-dimensional (2D) effects on plastic flow stability, a complete theoretical framework under plane strain state is established to model the 2D orthogonal cutting process. Based on the new framework, a set of governing equations with three dimensionless parameters are used to analytically derive a universal instability criterion, the approximate velocity fields, and stress fields in the expanding chip formation zone (CFZ). It is shown that the plastic flow of continuous chip may become unstable once the cutting speed reaches a critical value. In contrast to the shear localization deformation in the serrated chip, we found a new instability mechanism occurring in the continuous chip which undergoes the uniform but severe shear deformation due to the plane strain loadings. A new dimensionless parameter therefore is proposed to describe the plastic instability in continuous chip and the shear banding instability in serrated chip. The difference of two instability modes is further investigated in terms of dissipation mechanism of cutting energy, and the plastic instability of continuous chip is shown as the best instability mode regarding tool vibration and surface machining quality. These findings provide practical insights into improving modern cutting technology by controlling the plastic flow instability.
\end{abstract}

Keywords Metal cutting · Plastic flow stability · Instability criterion · Dimensional analysis · Transition of chip morphology · Cutting energy

\section{Introduction}

Despite metal machining serves as a very conventional technique widely applied in engineering, there are still plenty of basic science issues remaining unsolved [1,2]. Over the past two decades, the intensive efforts of engineers have been dedicated to improving machining process by enhancing machining quality, improving the efficiency, and increasing material hardness of tool and its service life. These engineering issues are directly related to the optimization of machining processing and the improvement of mechanical equipment. From the viewpoint of academic

Wei Ma

watwm@imech.ac.cn

Fei Shuang

shuangfei@ufl.edu

1 Institute of Mechanics, Chinese Academy of Sciences, Beijing 100190, China

2 Department of mechanical and Aerospace Engineering, University of Florida, Gainesville, FL 32611-6250, USA research, on the other hand, physicists are more concerned with the essence of cutting phenomenon and the underlying mechanisms. For example, what factors and mechanisms affect the material formability and workability? What is the dissipation mechanism of cutting energy? What are the mechanisms of material removal and chip formation? What factors determine the integrity of machined surface? So far, these questions have not been thoroughly clarified and are still the major interests in academic fields. One can see that these questions are closely related to some fundamental physical and mechanical phenomena, i.e., the plastic flow of chip materials and its instability, mechanical vibration, and non-linear dynamics as well as heat conduction and convection. Therefore, the study on these questions is of great importance for better understanding metal cutting process and developing the modern machining technique. In this context, we will focus on the plastic flow of chip materials and relevant instability modes in the OCP.

Recently, a lot of impressive achievements have been accomplished about the metal cutting mechanism for the common metals $[3,4]$. The emerging new materials such as alloy 
series of titanium and nickel, however, still face challenges in the machining process due to their difficult-cut properties [5]. These challenges arise many basic scientific problems on the mechanisms of metal cutting, for example, the chip formation mechanism [6-8], material removing mechanism [9], machined surface integrity $[10,11]$, fracture and cutting energy dissipation $[12,13]$, and cutting vibration behavior [14] and tool life $[15,16]$. One can see that these issues are closely related to the plastic flow of chip materials, the cutting conditions, and tool geometry. As one of the most commonly seen phenomena, the periodic shear banding in serrated chip can induce oscillation of stress, strain, and temperature and deteriorate the machined surface integrity and shorten the service life $[17,18]$. Previous studies on the cutting mechanism of Ti6Al4V alloy show that plastic instability of chip materials may change the dissipation mechanism of cutting energy and further affect the machining quality [19]. Nonetheless, the higher accuracy and efficiency is always our goal in the metal machining process [20], which has become a fundamental issue for both academic and engineering fields.

In contrast to the practical turning, the deformation of work materials in the OCP can be treated as the plane strain state instead of fully three-dimensional, which makes it easier to reveal the physical mechanisms. The orthogonal cutting model (OCM) is therefore widely used in the study of metal cutting. Especially, when investigating the plastic flow instability caused by shear bands in serrated chip, onedimensional OCM is often adopted and the deformation of work materials is assumed as uniform simple shear for the sake of simplification [21-23]. In this way, it is difficult to consider the influence of $2 \mathrm{D}$ effects such as the pressure and the friction at the rale face on the chip formation and material removal mechanisms.

In this work, we intend to study the influence of $2 \mathrm{D}$ effect in the OCP [19, 24]. First, we establish a 2D physical OCM and the corresponding mathematical descriptions in the framework of continuum mechanics. To evaluate the plastic flow stability of chip materials, a universal criterion as a necessary condition is then obtained by the linear stability analysis. Furthermore, a sufficient and necessary condition is proposed in terms of the true stress-strain relationship of chip materials. Either in the situation of complex stress loading or the simple stress loading, the criterion established in such manner can be used to determine the onset and evolution of plastic instability of materials. Through studying the plastic flow stability of chip materials for four kinds of metals in cutting process, we found two plastic instability modes of plastic flow in the OCP, which are closely related to the properties of work material and the cutting conditions. Therefore, this study allows us to have a better understanding for the mechanisms of chip formation and material removal in metal machining.

\section{Experiment}

The experiments performed here simulate the metal OCP in a wide range of cutting speeds. When the cutting speed is below $50 \mathrm{~m} / \mathrm{s}$, the Hopkinson pressure bar device is used to generate high-speed impacting loadings. When the cutting speed is in the range from 50 to $350 \mathrm{~m} / \mathrm{s}$, one-class light gas gun device is used to perform the ultra-high-speed cutting.

To carry out the high-speed cutting experiments, a selfdeveloped tentative platform was firstly set up with oneclass light gas gun. As shown in Fig. 1, it is made up of a double of baseplates, two tool anvils, tool setscrews, microcaliper, chip pool, projectile, launch tube, and laser speed transmitter. The baseplate can be adjusted in the vertical direction to control the horizontal degree of the whole cutting platform. Two anvils with large-mass of about $2 \times 8 \mathrm{~kg}$ are used to ensure the platform stability in high-speed cutting. The micro-calipers can be adjusted finely to precisely control the cutting thickness above $30 \mu \mathrm{m}$. The projectile consists of the specimen, specimen clamp, and bullet holder with total mass about $2.5 \mathrm{~kg}$, which can produce sufficiently large kinetic energy in the cutting process. When the cut thickness is less than $200 \mu \mathrm{m}$, the energy consumed in the material removal process within the cutting distance of $60 \mathrm{~mm}$ is much less than the kinetic energy of the projectile with high speed, so that the specimen can be assumed to be uniformly cut at a constant speed. The laser speed transmitter is used to measure the cutting speed. The chip samples are collected in the chip pools after cutting for the further microscopic observation. More details on the cutting experiments are described in the references [17, 19].

Four kinds of metals, Ti6Al4V alloy, Al7075 alloy, AISI 4340 steel, and AISI 1045 steel, are chosen as the testing materials to study the influence of material properties on the instability mechanisms. The chemical compositions of each metal are given in Table 1 and corresponding heat treatment

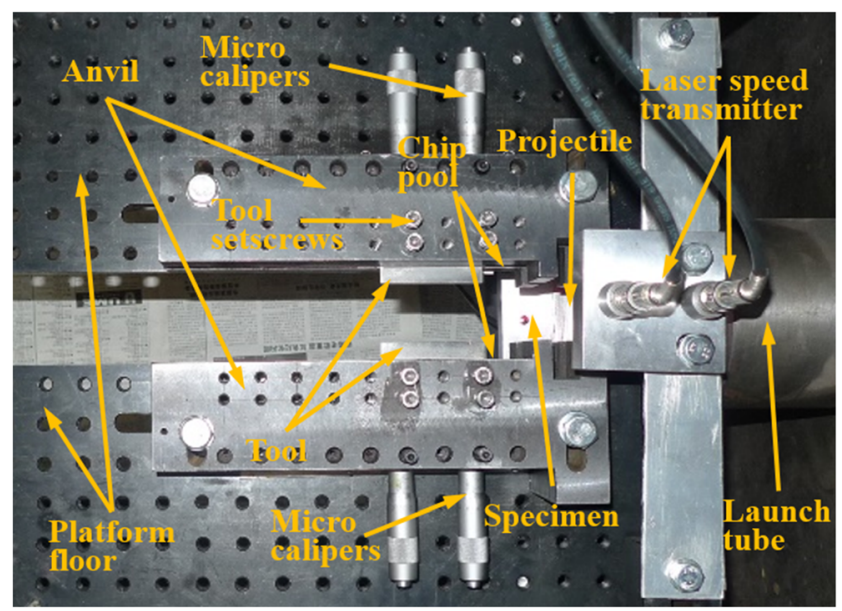

Fig. 1 The platform for carrying out the experiments of orthogonal cutting process of metals 
Table 1 The chemical composition of four testing metals

\begin{tabular}{lllllllll}
\hline \multirow{2}{*}{ Ti6Al4V [25] } & Ti & $\mathrm{Al}$ & $\mathrm{V}$ & $\mathrm{O}$ & $\mathrm{Fe}$ & $\mathrm{C}$ & $\mathrm{N}$ & $\mathrm{H}$ \\
& Balance & 6.5 & 4.25 & 0.16 & 0.04 & 0.02 & 0.015 & 0.0018 \\
\hline \multirow{2}{*}{ AISI 4340 [26] } & $\mathrm{Fe}$ & $\mathrm{C}$ & $\mathrm{Cr}$ & $\mathrm{P}$ & $\mathrm{Mo}$ & $\mathrm{Si}$ & $\mathrm{Cu}$ & $\mathrm{Mn}$ \\
& Balance & 0.405 & 0.851 & 0.011 & 0.233 & 0.248 & 0.178 & 0.696 \\
AISI 1045 [13] & Fe & $\mathrm{C}$ & $\mathrm{Si}$ & $\mathrm{Mn}$ & $\mathrm{P}$ & $\mathrm{S}$ & $\mathrm{Cr}$ & $\mathrm{Ni}$ \\
& Balance & 0.46 & 0.27 & 0.34 & 0.04 & 0.04 & 0.25 & 0.25 \\
& $\mathrm{Al}$ & $\mathrm{Zn}$ & $\mathrm{Mg}$ & $\mathrm{Cu}$ & $\mathrm{Cr}$ & $\mathrm{Fe}$ & $\mathrm{Si}$ & \\
& Balance & 5.6 & 2.5 & 1.6 & 0.23 & 0.5 & 0.4 & \\
\hline
\end{tabular}

conditions can be found in references [13, 25-27]. Assume that the plastic flow of materials is governed by $J_{2}$ flow law and complies with the Johnson-Cook (J-C) constitutive model [28]:

$\sigma_{\mathrm{eq}}=\left(1+B_{0} \varepsilon_{\mathrm{eq}}^{n}\right)\left(1+C_{0} \ln \dot{\varepsilon}_{\mathrm{eq}}\right)\left[1-\left(T-T_{0}\right)^{m}\right]$,

where $\sigma_{\mathrm{eq}}$ is the equivalent plastic stress, $\varepsilon_{\mathrm{eq}}^{n}$ the equivalent plastic strain, $\dot{\varepsilon}_{\mathrm{eq}}^{p}$ the plastic strain rate, and $\dot{\varepsilon}_{0}$ the reference strain rate. $T$ is the current temperature and $T_{0}$ the room temperature. $A_{0}, B_{0}, C_{0}, m$, and $n$ are J-C constitution model properties. The expression of material model in Eq. (1) has been processed by non-dimensionalization by using the group of basic dimensionless quantities in Eq. (2) below. The J-C model takes into account of the effects of strain hardening, rate sensitivity, and thermal softening on the plastic flow. Material failure due to the internal damage has been incorporated in the simulations of chip formation in terms of the J-C fracture model. The material properties and constitutive parameters are given in Table 2. The dimension of each material specimen is $60 \times 40$ $\times 4 \mathrm{~mm}$. The cut width and thickness are $4 \mathrm{~mm}$ and $100 \mu \mathrm{m}$, respectively. An uncoated tool of cemented carbide material is used in the dry machining process. The tool was designed with tip radius less than $20 \mu \mathrm{m}$, width $8 \mathrm{~mm}$, and a vanishing rake angle and clearance angle of $5^{\circ}$. As a consequence, the plastic deformation of workpiece and chip materials in the cutting process can be considered as an ideal plane strain state.

Figures 2 and 3 show the observations of scanning electron microscopic (SEM) images for the collected chip samples of different metals. A notable phenomenon is that the continuous chips transform into the serrated ones as the cutting speed increases (Fig. 2). Obviously, the transition speeds of chip morphology are related to the metal type. Though it is difficult to accurately determine the speed based on the experimental observation, the coarse estimations can be made as $0.5 \mathrm{~m} / \mathrm{s}$ for Ti6Al4V alloy, $6 \mathrm{~m} / \mathrm{s}$ for Al 7075 alloy, $6.7 \mathrm{~m} / \mathrm{s}$ for AISI 4340 steel, and $39 \mathrm{~m} / \mathrm{s}$ for AISI 1045 steel. The continuous chip materials experience the severe shear uniform deformation during the low-speed cutting process, resulting in the complete grain refinement of chip materials. The evident shear localization deformation is observed in primary shear zone (PSZ) during the high-speed cutting process, leading to the formation of serrated chips. In contrast to low-speed cutting, evident grain refinement is merely limited within the shear bands in high-speed cutting.

For the Ti6Al4V alloy, the ultra-high-speed cutting experiments with the cutting speed in the range of 90 to $320 \mathrm{~m} / \mathrm{s}$ were completed. It shows that the serrated chip transforms into the debris with corresponding critical cutting speed in the range of 60 to $90 \mathrm{~m} / \mathrm{s}$. In addition, the thickness of debris evidently decreases as the cutting speed increases (Fig. 3ac), implying that the shear banding spacing decreases as the cutting speed increases. Importantly, when the cutting speed is above $210 \mathrm{~m} / \mathrm{s}$ but less than $300 \mathrm{~m} / \mathrm{s}$, there exists another critical speed which corresponds to the morphology transition from the debris into continuous chips (Fig. 3d). This observation evidently demonstrates a tendency that the discontinuous chips may turn back to the continuous ones in the ultra-highspeed cutting process. The present experimental observation also confirms the previous numerical simulation results on high-speed cutting for Ti6Al4V alloy [9, 29, 30].

The shear localization deformation in PSZ is strongly dependent on the thermophysical properties of work materials such as the heat conductivity, heat capacity per unit volume, and the cutting conditions like cutting speed, chip thickness and the loading rate. Figure $4 \mathrm{a}$ plots the transition speeds from continuous to serrated with respect to the thermophysical properties of materials (as represented with the red curve and hollow points), which shows an increasing tendency as the ratio of the heat conductivity to the heat capacity per unit volume increases. The relation obeys an upward concave exponential function, demonstrating that good thermal conductivity and small heat capacity per unit volume can delay the transition of chip morphology through preventing shear banding instability. To further exam the influence of inertia effect, the transition speeds of four metals with respect to two dimensionless parameters are plotted in Fig. 4b, which illustrates the dependence of the speeds on the production of dimensionless parameters Re and $R_{c}$ (defined in following Eq. (8)). A positive relation with upward convex shape indicates that the inertia effect can restrain the nucleation and evolution of shear bands. 
Table 2 The material properties and the J-C constitutive model parameters of the four testing metals

\begin{tabular}{|c|c|c|c|c|}
\hline & Ti6Al4V [25] & AISI 4340 [26] & AISI 1045 [13] & Al 7075 [27] \\
\hline Density $\rho\left(\mathrm{kg} / \mathrm{m}^{3}\right)$ & 4420 & 7830 & 7850 & 2770 \\
\hline Elastic modulus $E(\mathrm{GPa})$ & 114 & 200 & 210 & 71 \\
\hline Poisson's ratio $\nu$ & 0.342 & 0.29 & 0.3 & 0.3 \\
\hline Specific heat $c(\mathrm{~J} /(\mathrm{kg} \mathrm{K}))$ & 560 & 477 & 452 & 885 \\
\hline Thermal conductivity $\lambda(\mathrm{W} /(\mathrm{m} \mathrm{K}))$ & 7.2 & 38 & 50 & 130 \\
\hline Expansion coefficient $\alpha\left(\mathrm{K}^{-1}\right)$ & $9.2 \times 10^{-6}$ & $3.2 \times 10^{-5}$ & $1.5 \times 10^{-5}$ & $2.32 \times 10^{-5}$ \\
\hline Melting temperature $T_{m}(\mathrm{~K})$ & 1930 & 1793 & 1765 & 893 \\
\hline Taylor-Quinney coefficient $\beta$ & 0.9 & 0.93 & 0.93 & 0.9 \\
\hline Shear failure strain $\gamma_{c}$ & 0.28 & 0.22 & 0.22 & 0.05 \\
\hline \multicolumn{5}{|l|}{$\mathrm{J}-\mathrm{C}$ constitution parameters } \\
\hline$A_{0}(\mathrm{MPa})$ & 782 & 792 & 496 & 546 \\
\hline$B_{0}(\mathrm{MPa})$ & 498 & 510 & 434 & 678 \\
\hline$n$ & 0.28 & 0.26 & 0.307 & 0.71 \\
\hline$C_{0}$ & 0.028 & 0.014 & 0.047 & 0.024 \\
\hline$m$ & 1 & 1.03 & 0.804 & 1.56 \\
\hline$\dot{\varepsilon}_{0}(1 / \mathrm{s})$ & 1 & 1 & 1 & 1 \\
\hline
\end{tabular}

\section{Theory}

\subsection{Theoretical model}

In the OCP of metals, the ratio of workpiece width to chip thickness is sufficiently large so that the deformation of work materials can be treated as a plane strain state. In general, 1D OCM focuses on the shear localization deformation in PSZ and the effect of shear stress on the shear plane is considered as the dominating mechanism accounting for the formation of serrated chips. For instance, the shear plane model [6] (Fig. 5a) and the card model [21] (Fig. 5b) assumed that the chip velocity on the shear plane is
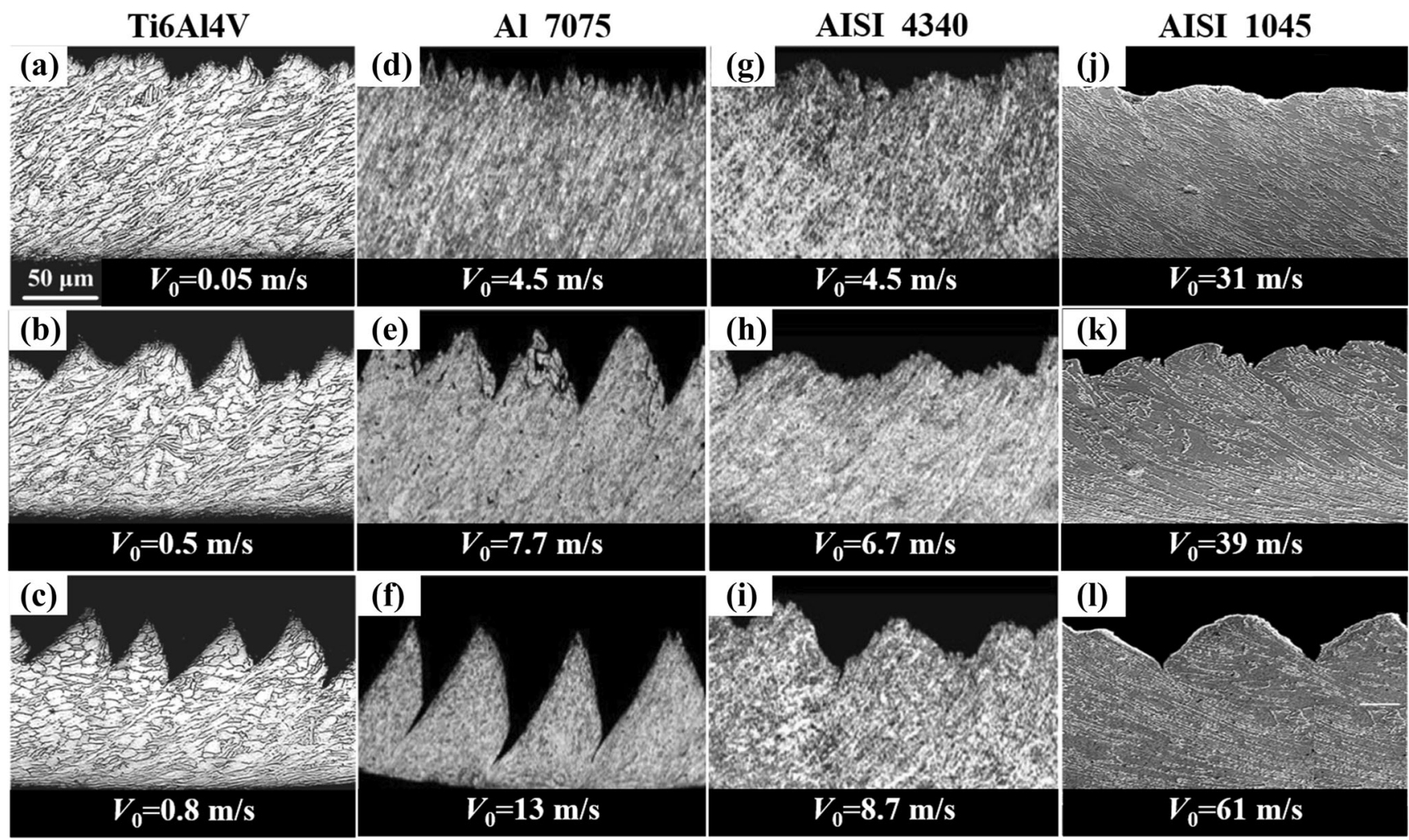

Fig. 2 The SEM observation of the cross-section of chip samples of the four testing metals 
(a)

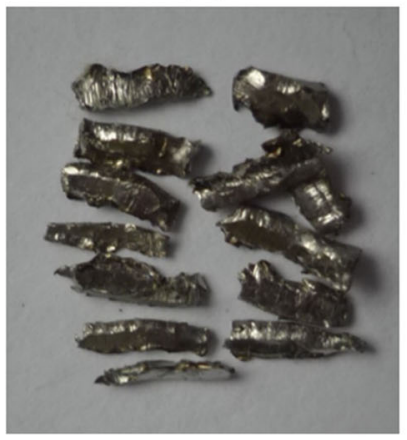

$V_{0}=90 \mathrm{~m} / \mathrm{s}$ (b)

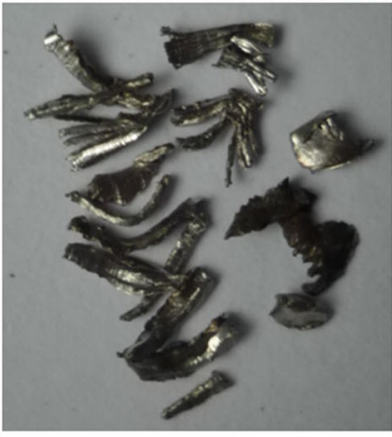

$V_{0}=170 \mathrm{~m} / \mathrm{s}$ (c)

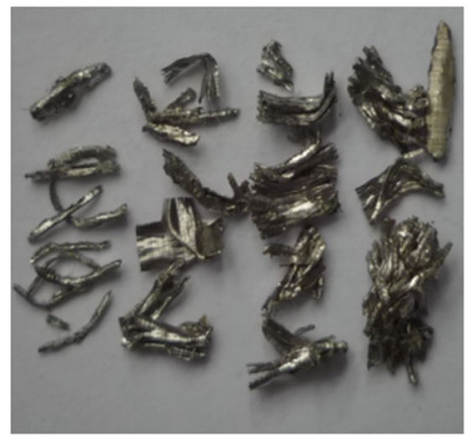

$V_{0}=210 \mathrm{~m} / \mathrm{s}$ (d)

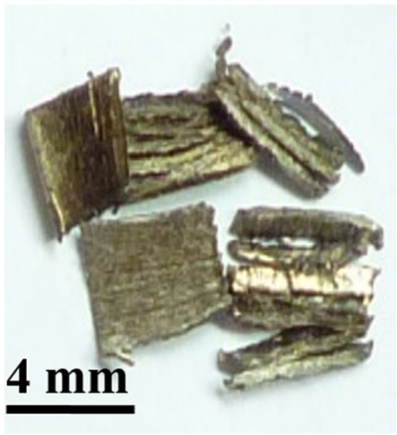

$V_{0}=317 \mathrm{~m} / \mathrm{s}$

Fig. 3 a-d The SEM observations of the Ti6Al4V alloy chips obtained in the high-speed cutting process

discontinuous. These simple models allow us to have the basic understanding of the relationship between cutting forces and geometric characteristics of cutting process as well as the
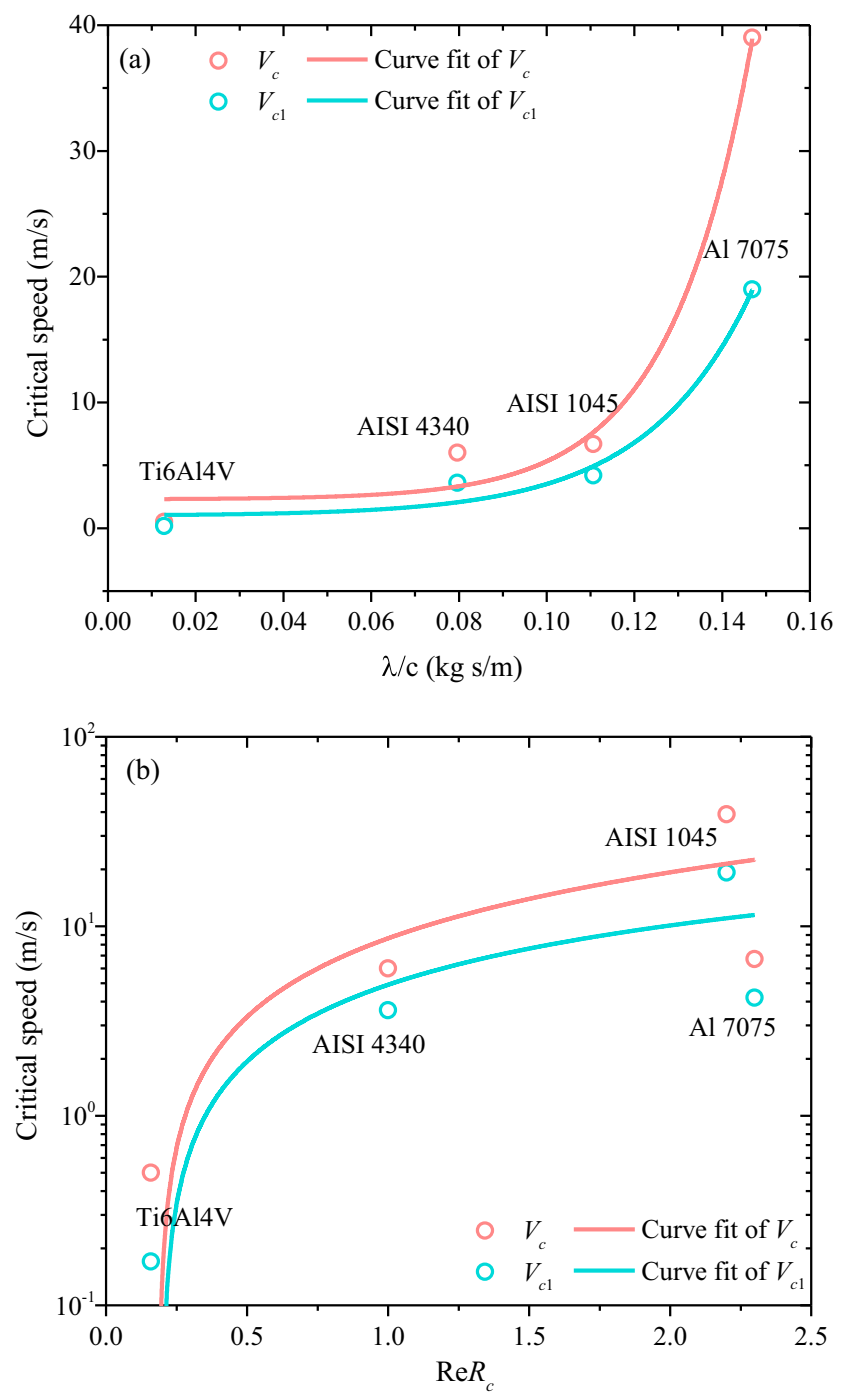

Fig. 4 The dependences of the critical speeds of plastic instability of chip flow on the thermophysical properties (a) and inertial (b) of materials preliminary mechanism of chip deformation, even though such assumption contradicts the experimental observation that the cutting velocity is continuous in the PSZ [22]. Through considering the thickness of PSZ, however, the parallel-sided shear zone model [23] (Fig. 5c) describes not only the continuity of cutting speed, but also the shear localization deformation in PSZ, which can be used to study the formation mechanism of serrated chip in high-speed cutting.

Unlike the 1D OCM, the 2D OCM considers the plastic flow of chip materials and its instability behavior in an extensive chip formation zone (CFZ) labeled with parallelogram $O A B C$ which includes the PSZ and the secondary shear zone (SSZ) (Fig. 5d) [24, 31]. This model also considers the shear banding growth in the shear plane and the matter flux along the rake surface. The shear stress $\sigma_{12}$ on the shear plane and the distribution pressure on the rake surface $p_{N}$ jointly cause the plastic flow of chip material. The contact between tool and chip obeys the Coulomb friction law with a constant dry friction coefficient. The cutting conditions include the cutting speed, cutting thickness, and the rake angle [19].

To clearly reveal the main factors affecting the metal cutting process, a mathematical formulation in dimensionless form is established based on the 2D OCM. The cutting speed $V_{0}$, cutting thickness $h_{0}$, and the difference between melting and room temperature $T_{\mathrm{m}}-T_{0}$ are chosen as the basic unit quantities to normalize all quantities. Afterwards, the dimensionless time, the Cartesian coordinates, velocity, stress, and temperature of material point in the reference configuration can be written as

$$
\begin{aligned}
\hat{t} & =\frac{V_{0} t}{h_{0}}, \hat{x}_{1}=\frac{x_{1}}{h_{0}}, \hat{x_{2}}=\operatorname{Re} \frac{x_{2}}{h_{0}}, \hat{v}_{1}=\frac{v_{1}}{V_{0}}, \hat{v}_{2}=\operatorname{Re} \frac{v_{2}}{V_{0}}, \hat{\sigma}_{i j} \\
& =\frac{\sigma_{i j}}{A_{0}}, \hat{\hat{i}_{i j}}=\frac{\varepsilon_{i j}}{\dot{\varepsilon}_{0}}, \hat{T}=\frac{T}{T_{\mathrm{m}}-T_{0}},
\end{aligned}
$$


Fig. 5 Three 1D OCMs (a-c) widely used in material cutting process and the 2D OCM (d) used in the present study of material cutting process
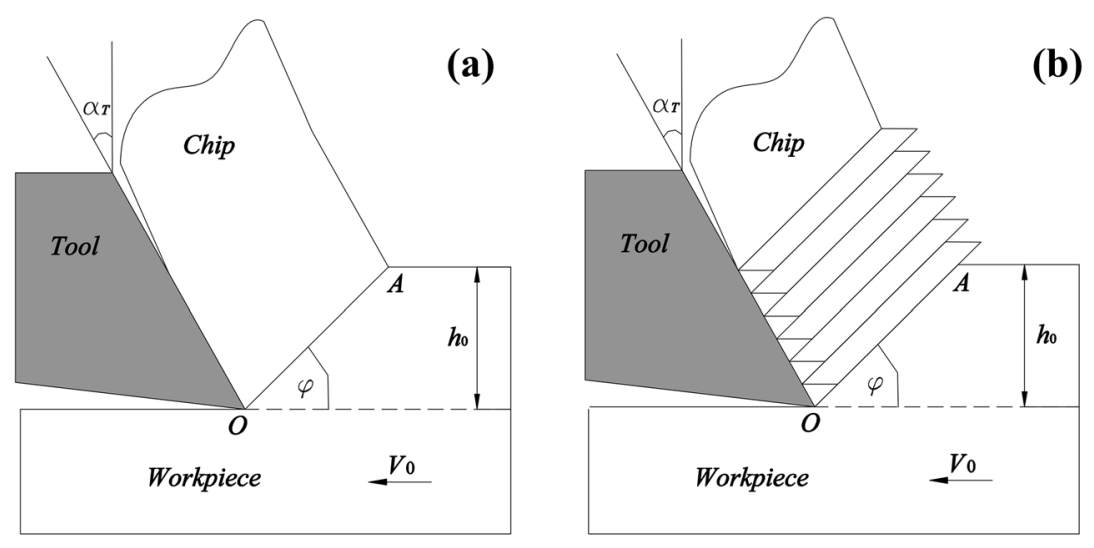

(c)

(d)
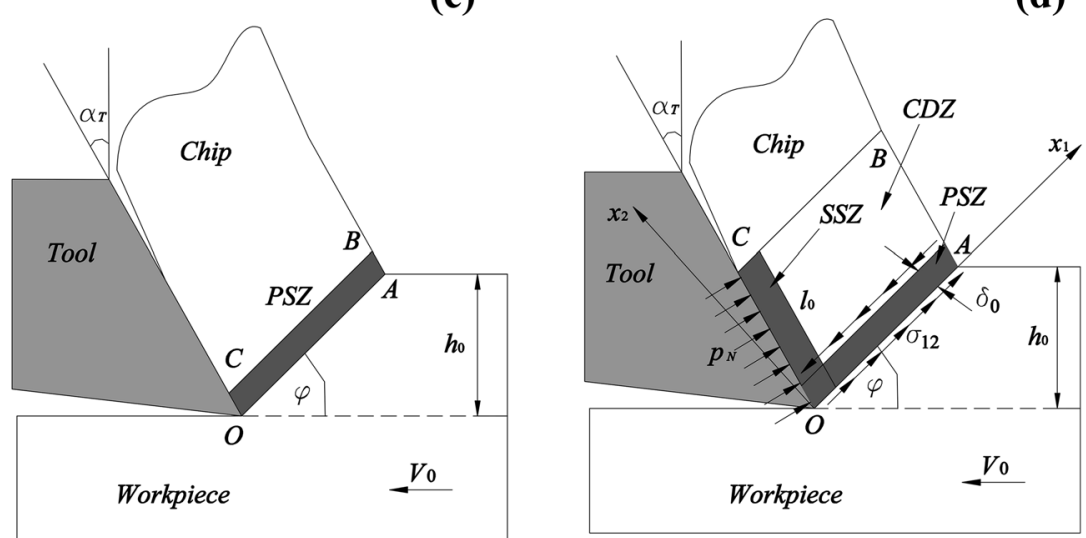

where the top mark "^" denotes that the correlation quantity is dimensionless. For convenience, the symbol "^" is omitted in below context. Re is a dimensionless parameter to be determined in the non-dimensionalization process of basic equations. After the normalization of the basic governing equations by using the basic quantities in Eq. (2), we have energy balance equation:

$$
\begin{aligned}
\frac{\partial T}{\partial t}= & R_{S}\left(\frac{\partial^{2} T}{\partial x_{1}^{2}}+\operatorname{Re}^{2} \frac{\partial^{2} T}{\partial x_{2}^{2}}\right) \\
& +R_{c}\left(\sigma_{11} \frac{\partial \varepsilon_{11}}{\partial t}+\sigma_{22} \frac{\partial \varepsilon_{22}}{\partial t}+2 \sigma_{12} \frac{\partial \varepsilon_{12}}{\partial t}\right),
\end{aligned}
$$

momentum balance equation:

$$
\left\{\begin{array}{l}
\frac{\partial v_{1}}{\partial t}+v_{1} \frac{\partial v_{1}}{\partial x_{1}}+v_{2} \frac{\partial v_{1}}{\partial x_{2}}=\frac{1}{\operatorname{Re}} \frac{\partial \sigma_{11}}{\partial x_{1}}+\frac{\partial \sigma_{12}}{\partial x_{2}} \\
\frac{\partial v_{2}}{\partial t}+v_{1} \frac{\partial v_{2}}{\partial x_{1}}+v_{2} \frac{\partial v_{2}}{\partial x_{2}}=\frac{\partial \sigma_{12}}{\partial x_{1}}+\operatorname{Re} \frac{\partial \sigma_{22}}{\partial x_{2}}
\end{array},\right.
$$

compatibility condition of deformation:

$\frac{\partial^{2} \varepsilon_{1}}{\partial x_{2}^{2}}+\frac{\partial^{2} \varepsilon_{2}}{\partial x_{1}^{2}}-2 \frac{\partial^{2} \varepsilon_{12}}{\partial x_{1} \partial x_{2}}=0$, and continuity equation:

$\frac{\partial v_{1}}{\partial x_{1}}+\frac{\partial v_{2}}{\partial x_{2}}=0$

The dimensionless strain and strain rate components:

$$
\begin{aligned}
& \varepsilon_{11}=\frac{\partial u_{1}}{\partial x_{1}}, \varepsilon_{22}=\frac{\partial u_{2}}{\partial x_{2}}, \varepsilon_{12}=\frac{1}{2}\left(\operatorname{Re} \frac{\partial u_{1}}{\partial x_{2}}+\frac{1}{\operatorname{Re}} \frac{\partial u_{2}}{\partial x_{1}}\right) \\
& \dot{\varepsilon}_{11}=\frac{\partial v_{1}}{\partial x_{1}}, \dot{\varepsilon}_{22}=\frac{\partial v_{2}}{\partial x_{2}}, \dot{\varepsilon}_{12}=\frac{1}{2}\left(\operatorname{Re} \frac{\partial v_{1}}{\partial x_{2}}+\frac{1}{\operatorname{Re}} \frac{\partial v_{2}}{\partial x_{1}}\right) .
\end{aligned}
$$

In Eqs. (3)-(7), three dimensionless parameters are founds as:

$\operatorname{Re}=\frac{\chi\left(V_{0}\right) \rho V_{0}^{2}}{A_{0}}, R_{c}=\frac{\lambda}{\rho c h_{0} V_{0}}, R_{s}=\frac{\beta A_{0}}{\rho c\left(T_{\mathrm{m}}-T_{0}\right)}$,

where $\rho, c$, and $\lambda$ are the mass density, heat capacity per unit volume, and thermal conductivity of work material, respectively. In Eq. (8), the parameter Re, like the Reynolds number in fluid mechanics, is the ratio of inertia forces to viscous forces; here, the viscous force is the initial yield strength of material $A_{0}$. The parameter $R_{c}$, named the Reynolds thermal number [32], is the ratio of the heat conduction to the workpiece and the heat convection from the tool by chip motion. The parameter $R_{S}$ depends 
on Taylor-Quinny coefficient $\beta$, which denotes the effect of thermal power conversion during plastic deformation.

Although Eqs. (3)-(6) defined in 2D space are capable of describing the plastic flow of chip material in the plane zone $O A B C$, it is inconvenient when dealing with the shear banding instability since the shear banding nucleation and evolution are usually treated as 1D simple shear flow process. We note that, as $\mathrm{Re} \gg 1$, for example $\mathrm{Re}=10$, the $2 \mathrm{D}$ equations can be simplified as 1D Prandtl boundary layer equation which was used to study the shear banding instability of material [33]. The question is if the 2D equations can describe the plastic flow of continuous chip and the shear banding instability behavior simultaneously. Here, we found the answer depends on the value of the parameter Re. The experimental results in Fig. 2 demonstrate that the transition speeds of chip morphology from continuous to serrated are generally in the order of magnitude $10^{-1}$ to $10^{1} \mathrm{~m} / \mathrm{s}$, which corresponds to the very small Re. $\operatorname{Re} \gg 1$ (we choose $\mathrm{Re}=10$ in this paper) therefore generally represents the very high cutting speed comparing with the actual transition speed. To use $\mathrm{Re}=10$ as the general condition indicating the chip morphology transition from continuous to serrated, a function $\chi\left(V_{0}\right)$ is introduced to modify $\mathrm{Re}$ as given in Eq. (8). Based on the cutting experimental results in Figs. 2 and 3, the function $\chi\left(V_{0}\right)$ takes the form

$$
\begin{aligned}
& \chi\left(V_{0}\right) \\
& = \begin{cases}\frac{A_{0}}{\rho V_{0 i}^{2}}\left(\operatorname{Re}_{c}+\operatorname{Re}_{0} \frac{V_{0 i}^{2}-V_{0}^{2}}{V_{0}^{2}}\right) & \left(\operatorname{Re}_{0} \leq \operatorname{Re}<\operatorname{Re}_{c}\right) \\
\frac{A_{0} \operatorname{Re}_{c}}{\rho V_{0}^{2}}-\left(1-\frac{V_{0 i}}{V_{0}}\right)\left(1-\frac{V_{0 T}}{V_{0}}\right) & \left(\operatorname{Re} \geq \operatorname{Re}_{c}\right) \\
\frac{A_{0}}{\rho V_{0 T}^{2}}\left(\operatorname{Re}_{c}+\frac{V_{0 T}^{2}-\operatorname{Re}_{c} V_{0 T 1}^{2}}{V_{0 T}^{2}-V_{0 T 1}^{2}} \frac{V_{0 T}^{2}-V_{0}^{2}}{V_{0}^{2}}\right) & \left(\operatorname{Re}_{c}>\operatorname{Re} \geq \operatorname{Re}_{01}\right)\end{cases}
\end{aligned}
$$

It can be seen that the function $\chi\left(V_{0}\right)$ is related to the cutting conditions and workpiece material properties. $\mathrm{Re}_{0}=1$ corresponds to the initial cutting speed $V_{0}=0$ and $\operatorname{Re}_{c}=10$ the transition speed $V_{0 i}$ from continuous to serrated. The speed $V_{0 T}$ denotes the transition from serrated to continuous in ultrahigh-speed cutting. Figure 6 illustrates the original and modified parameter Re with respect to the cutting speeds. The original Re without modified function $\chi\left(V_{0}\right)$ (represented by the green line in the figure) is always a small quantity even when the cutting speed approaches to $350 \mathrm{~m} / \mathrm{s}$, while the modified $\mathrm{Re}$ is equal to or larger than 10 once the cutting speed exceeds the transition speed $V_{0 i}$. For each testing metal, the condition $\mathrm{Re}=\mathrm{Re}_{c}=10$ determines two transition speeds, which correspond to the transition from continuous into serrated at $V_{0}=V_{0 i}$ and from serrated into continuous at $V_{0}=V_{0 T}$. Accordingly, the 2D equations can be simplified as 1D Prandtl boundary layer equation when $\operatorname{Re}>\operatorname{Re}_{c}$. By modifying the original parameter Re with function $\chi\left(V_{0}\right)$ in Eqs. (3)-(6), the 2D equations can describe the plastic flow stability of

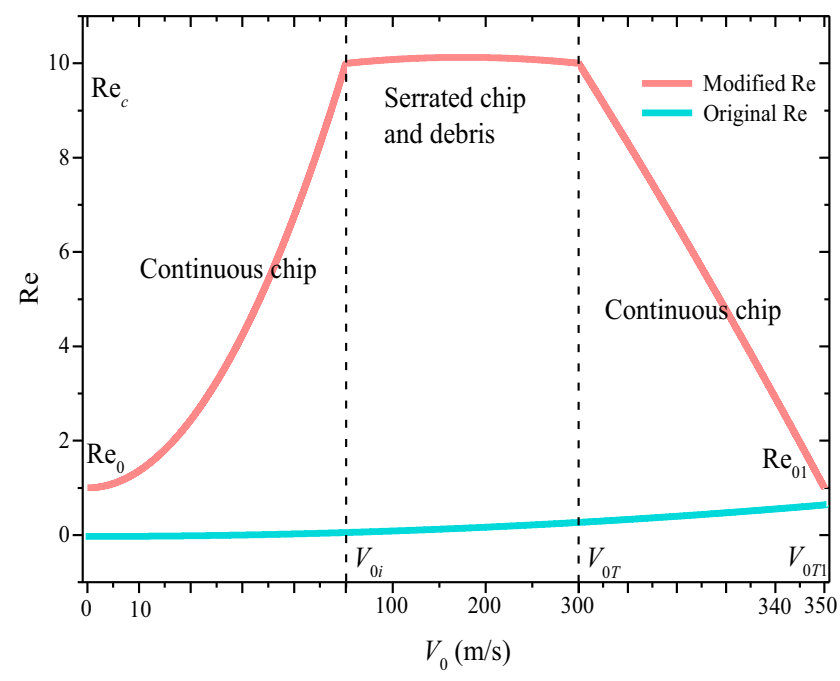

Fig. 6 The relationship curves of the corrected and uncorrected Reynolds number with the cutting speeds

continuous chip in the plane strain state when $\operatorname{Re}_{0}<\operatorname{Re}<$ $\mathrm{Re}_{c}$, and also can describe the shear band instability when the serrated chip develops when $\operatorname{Re} \geq \operatorname{Re}_{c}$ as well as the plastic flow process of the continuous chip in ultra-high-speed cutting when $\operatorname{Re}_{c}>\operatorname{Re}>\operatorname{Re}_{01}$. Therefore, the 2D Eqs. (3)-(6) can describe not just the plastic flow of chip material in the plane strain state, but also the shear band instability. More importantly, two morphology transitions can be captured, which can help us understand the underlying mechanisms in a unified framework.

\subsection{Instability condition}

As stated above, when $\operatorname{Re}_{0}<\operatorname{Re}<\operatorname{Re}_{c}$ and $\operatorname{Re}_{c}>\operatorname{Re}>$ $\mathrm{Re}_{01}$, Eqs. (3)-(6) along with the constitutive relation Eq. (1) describe the plastic deformation of continuous chip in a plane strain state; when $\operatorname{Re} \geq \operatorname{Re}_{c}$, these equations describe the shear banding instability and the formation of serrated chips and debris; $\mathrm{Re}=\mathrm{Re}_{c}$ denotes the critical condition of chip morphology transition. To better understand the plastic flow process of material in 2D OCP and further predict the transition behavior, it is necessary to establish a universal and practical criterion on the basis of material properties. Linear stability analysis has been successfully applied to evaluate the stability of material plastic flow in cutting process. Considering the response of a homogeneously deformed thermoviscoplastic solid subjected to small disturbances, we assume the inhomogeneous solutions of Eqs. (3)-(6) have the form

$\left\{\begin{array}{c}\varepsilon_{i j} \\ \sigma_{i j} \\ \varepsilon_{i j} \\ v_{i} \\ T\end{array}\right\}=\left\{\begin{array}{c}\varepsilon_{0 i j} \\ \sigma_{0 i j} \\ \varepsilon_{0 i j} \\ v_{0 i} \\ T_{0}\end{array}\right\}+\left\{\begin{array}{c}\delta \varepsilon_{i j} \\ \delta \sigma_{i j} \\ \delta \varepsilon_{i j} \\ \delta v_{i} \\ \delta T\end{array}\right\} \exp [\omega t+i(\mathbf{k} \cdot \mathbf{x})]$ and $\left\{\begin{array}{c}\varepsilon_{0 i j} \\ \sigma_{0 i j} \\ \varepsilon_{0 i j} \\ v_{0 i} \\ T_{0}\end{array}\right\} \geq\left\{\begin{array}{c}\delta \varepsilon_{i j} \\ \delta \sigma_{i j} \\ \delta \varepsilon_{i j} \\ \delta v_{i} \\ \delta T\end{array}\right\}$ 
where $\left\{{ }_{i j}\right\}(i, j=1,2)$ represents a group of inhomogeneous solution of Eqs. (3)-(6), $\left\{\cdot_{0 i j}\right\}$ the corresponding homogeneous solution. $\left\{\delta \bullet_{i j}\right\}$ is a small constant characterizing the amplitude of perturbation of $\left\{\cdot_{0 i j}\right\}$. The dimensionless wave vector is defined as $\mathbf{k}=k_{1} \mathbf{n}_{1}+k_{2} \mathbf{n}_{2}$ with $k^{2}=$ $k_{1}{ }^{2}+k_{2}{ }^{2}$. Inserting Eq. (10) into Eq. (1) and Eqs. (3)-(6) and considering only the first order perturbation terms, the characteristic equation with regard to the perturbation growth rate $\omega$ can be obtained as:

$J_{1} \omega^{3}+J_{2} \omega^{2}+J_{3} \omega+J_{4}=0$ where the four coefficients $J_{1}, J_{2}, J_{3}$, and $J_{4}$ have the form

$$
\left\{\begin{array}{l}
J_{1}=2 \operatorname{Re}\left(\varsigma_{2}^{2} \eta_{11}+\varsigma_{1}^{2} \eta_{22}\right) \\
J_{2}=2 \operatorname{Re}\left(\varsigma_{2}^{2} \eta_{11}+\varsigma_{1}^{2} \eta_{22}\right)\left(R_{s} k^{2}-A_{1}^{p} R_{c} P_{0}\right)+B_{2}^{r} R_{0} k^{2} \\
J_{3}=\left[B_{1} A_{2}^{p} R_{c} P_{0}+\left(B_{3}^{r} A_{2}^{p}-B_{2}^{r} A_{1}^{p}\right) R_{0} R_{c} P_{0}+B_{2}^{q} Q_{0}+B_{2}^{r} R_{s} k^{2} R_{0}\right] k^{2}, \\
J_{4}=\left[B_{2}^{q} R_{s} k^{2}+\left(B_{3}^{q} A_{2}^{p}-B_{2}^{q} A_{1}^{p}\right) R_{c} P_{0}\right] k^{2} Q_{0}
\end{array}\right.
$$

and

$A_{1}^{p}=\dot{\varepsilon}_{110} p_{11}+\dot{\varepsilon}_{220} p_{22}+2 \dot{\varepsilon}_{120} p_{12}$

$A_{2}^{p}=\varsigma_{1} \varsigma_{2}\left(p_{11}+p_{22}\right)+p_{12}$

$B_{1}=2 \eta_{12}\left(\varsigma_{2}^{2} \eta_{11}+\varsigma_{1}^{2} \eta_{22}\right) \sigma_{120}+2 \eta_{12} \varsigma_{1} \varsigma_{2}\left(\eta_{11} \sigma_{110}+\eta_{22} \sigma_{220}\right)$

$B_{2}^{q}=2 H_{21} \eta_{12}\left[\varsigma_{1} \varsigma_{2}\left(q_{1112}+q_{2212}\right)+q_{1212}\right]+$

$2 \eta_{12} \varsigma_{1} \varsigma_{2}\left(\eta_{11}\left[\varsigma_{1} \varsigma_{2}\left(q_{1111}+q_{2211}\right)+q_{1211}\right]+\eta_{22}\left[\varsigma_{1} \varsigma_{2}\left(q_{1122}+q_{2222}\right)+q_{1222}\right]\right)$

$B_{2}^{r}=2 H_{21} \eta_{12}\left[\varsigma_{1} \varsigma_{2}\left(r_{1112}+r_{2212}\right)+r_{1212}\right]+$

$2 \eta_{12} \varsigma_{1} \varsigma_{2}\left(\eta_{11}\left[\varsigma_{1} \varsigma_{2}\left(r_{1111}+r_{2211}\right)+r_{1211}\right]+\eta_{22}\left[\varsigma_{1} \varsigma_{2}\left(r_{1122}+r_{2222}\right)+r_{1222}\right]\right)$

$B_{3}^{q}=2 \eta_{12}\left(\varsigma_{2}^{2} \eta_{11}+\varsigma_{1}^{2} \eta_{22}\right)\left(\varepsilon_{110} q_{1112}+\varepsilon_{220} q_{2212}+2 \varepsilon_{120} q_{1212}\right)+$

$2 \eta_{12} \varsigma_{1} \varsigma_{2}\left[\eta_{11}\left(\varepsilon_{110} q_{1111}+\dot{\varepsilon}_{220} q_{2211}+2 \dot{\varepsilon}_{120} q_{1211}\right)+\eta_{22}\left(\dot{\varepsilon}_{110} q_{1122}+\dot{\varepsilon}_{220} q_{2222}+2 \dot{\varepsilon}_{120} q_{1222}\right)\right]$

$B_{3}^{r}=2 \eta_{12}\left(\varsigma_{2}^{2} \eta_{11}+\varsigma_{1}^{2} \eta_{22}\right)\left(\dot{\varepsilon}_{110} r_{1112}+\dot{\varepsilon}_{220} r_{2212}+2 \varepsilon_{120} r_{1212}\right)+$

$2 \eta_{12} \varsigma_{1} \varsigma_{2}\left[\eta_{11}\left(\varepsilon_{110} r_{1111}+\dot{\varepsilon}_{220} r_{2211}+2 \dot{\varepsilon}_{120} r_{1211}\right)+\eta_{22}\left(\dot{\varepsilon}_{110} r_{1122}+\dot{\varepsilon}_{220} r_{2222}+2 \dot{\varepsilon}_{120} r_{1222}\right)\right]$

$\varsigma_{i}=\frac{k_{i}}{\sqrt{k_{1}^{2}+k_{2}^{2}}}, \eta_{11}=\frac{\delta \varepsilon_{11}}{\delta e_{N}}, \eta_{22}=\frac{\delta \varepsilon_{22}}{\delta e_{N}}, \eta_{12}=\frac{\delta \varepsilon_{12}}{\delta e_{S}}, \delta e_{N}=\delta \varepsilon_{11}+\delta \varepsilon_{22}, \delta e_{S}=\delta \varepsilon_{12}$

$p_{i j}=\frac{P_{i j}}{P_{0}}, q_{i j k l}=\frac{Q_{i j k l}}{Q_{0}}, r_{i j k l}=\frac{R_{i j k l}}{R_{0}}$

$P_{0}=P_{11}+P_{22}+P_{12}$

$Q_{0}=Q_{1111}+Q_{1122}+Q_{1112}+Q_{2211}+Q_{2222}+Q_{2212}+Q_{1211}+Q_{1222}+Q_{1212}$

$R_{0}=R_{1111}+R_{1122}+R_{1112}+R_{2211}+R_{2222}+R_{2212}+R_{1211}+R_{1222}+R_{1212}$

$P_{i j}=\frac{\partial \sigma_{i j}}{\partial T}=-\frac{\sigma_{\mathrm{eq}} m\left(T-T_{0}\right)^{m-1}}{1-\left(T-T_{0}\right)^{m}}$

$Q_{i j k l}=\frac{\partial \sigma_{i j}}{\partial e_{k l}}=\frac{\left(21+\delta_{i j}\right) e_{k l} \sigma_{\mathrm{eq}}^{2}}{9 s_{i j} \varepsilon_{\mathrm{eq}}} \frac{C_{0} n\left(\varepsilon_{\mathrm{eq}}\right)^{n-1}}{1+B_{0}\left(\hat{\varepsilon}_{\mathrm{eq}}\right)^{n}}$

$R_{i j k l}=\frac{\partial \sigma_{i j}}{\partial \dot{e}_{i j}}=\frac{2\left(1+\delta_{k l}\right) \sigma_{\mathrm{eq}}^{2} \dot{e}_{i j}}{9 S_{k l} \dot{\varepsilon}_{\mathrm{eq}}^{2}} \frac{C_{0}}{1+C_{0} \ln \dot{\varepsilon}_{\mathrm{eq}}}$.

The dimensionless growth rate $\omega$ and the wave number $\mathbf{k}$ are defined by

$\omega \sim \frac{\omega_{0} h_{0}}{V_{0}}, \mathbf{k} \sim h_{0} \mathbf{k}_{\mathbf{0}}$.

The stability of chip flow is dependent on the growth rate $\omega$. If $\omega \leq 0$, the chip flow is stable; otherwise, plastic instability is possible since the plastic flow strain can continually grow with time. The negative terms in the coefficients $J_{2}$ and $J_{3}$ are related to the thermal softening factor of materials and may be the sources causing the plastic instability of chip flow. Moreover, the occurrence of plastic instability certainly conjoins with a set of wave numbers. By using the condition $d \omega / d k_{0}^{2}=0$, the criterion for evaluating the plastic flow stability of chip material in a plane strain state can be found as:

$F_{\text {Inst }}=\frac{\left(F_{1}+F_{2} R_{0}+F_{3} R_{c} Q_{0}\right) R_{s} P_{0}}{F_{4} Q_{0}}>1$, 
where

$$
\begin{aligned}
F_{1}= & 2 \eta_{12} \Pi^{1}\left[\varsigma_{1} \varsigma_{2}\left(\eta_{11} \sigma_{110}+\eta_{22} \sigma_{220}\right)+\operatorname{Re}\left(\varsigma_{2}^{2} \eta_{11}+\varsigma_{1}^{2} \eta_{22}\right) \sigma_{120}\right] \\
F_{2}= & 2 \eta_{12} \Pi^{1}\left[\varsigma_{1} \varsigma_{2}\left(\eta_{11} \Theta_{11}^{2}+\eta_{22} \Theta_{22}^{2}\right)+\operatorname{Re}\left(\varsigma_{2}^{2} \eta_{11}+\varsigma_{1}^{2} \eta_{22}\right) \Theta_{12}^{2}\right]- \\
& 2 \eta_{12} \Pi^{2}\left[\varsigma_{1} \varsigma_{2}\left(\eta_{11} \Theta_{11}^{1}+\eta_{22} \Theta_{22}^{1}\right)+\operatorname{Re}\left(\varsigma_{2}^{2} \eta_{11}+\varsigma_{1}^{2} \eta_{22}\right) \Theta_{12}^{1}\right] \\
F_{3}= & 2 \eta_{12} \Pi^{1}\left[\varsigma_{1} \varsigma_{2}\left(\eta_{11} \Xi_{11}^{2}+\eta_{22} \Xi_{22}^{2}\right)+\operatorname{Re}\left(\varsigma_{2}^{2} \eta_{11}+\varsigma_{1}^{2} \eta_{22}\right) \Xi_{12}^{2}\right]- \\
& 2 \eta_{12} \Pi^{2}\left[\varsigma_{1} \varsigma_{2}\left(\eta_{11} \Xi_{11}^{1}+\eta_{22} \Xi_{22}^{1}\right)+\operatorname{Re}\left(\varsigma_{2}^{2} \eta_{11}+\varsigma_{1}^{2} \eta_{22}\right) \Xi_{12}^{1}\right] \\
F_{4}= & 2 \eta_{12}\left[\varsigma_{1} \varsigma_{2}\left(\eta_{11} \Xi_{11}^{1}+\eta_{22} \Xi_{22}^{1}\right)+\operatorname{Re}\left(\varsigma_{2}^{2} \eta_{11}+\varsigma_{1}^{2} \eta_{22}+\eta_{12}\right) \Xi_{12}^{1}\right] \\
\Pi^{1}= & \varsigma_{1} \varsigma_{2}\left(p_{11}+p_{22}\right)+\left(\frac{\varsigma_{1}^{2}}{\operatorname{Re}^{2}}+\varsigma_{2}^{2}\right) p_{12}, \Pi^{2}=\dot{\varepsilon}_{110} p_{11}+\dot{\varepsilon}_{220} p_{22}+\frac{1}{2}\left(\operatorname{Re} \frac{\partial v_{10}}{\partial x_{2}}+\frac{1}{\operatorname{Re}} \frac{\partial v_{20}}{\partial x_{1}}\right) p_{12} \\
\Xi_{i j}^{1}= & \varsigma_{1} \varsigma_{2}\left(q_{11 i j}+q_{22 i j}\right)+\left(\frac{\varsigma_{1}^{2}}{\operatorname{Re}^{2}}+\varsigma_{2}^{2}\right) q_{12 i j}, \Xi_{i j}^{2}=\dot{\varepsilon}_{110} q_{11 i j}+\dot{\varepsilon}_{220} q_{22 i j}+\frac{1}{2}\left(\operatorname{Re} \frac{\partial v_{10}}{\partial x_{2}}+\frac{1}{\operatorname{Re}} \frac{\partial v_{20}}{\partial x_{1}}\right) q_{12 i j} \\
\Theta_{i j}^{1}= & \varsigma_{1} \varsigma_{2}\left(r_{11 i j}+r_{22 i j}\right)+\left(\frac{\varsigma_{1}^{2}}{\operatorname{Re}^{2}}+\varsigma_{2}^{2}\right) r_{12 i j}, \Theta_{i j}^{2}=\dot{\varepsilon}_{110} r_{11 i j}+\dot{\varepsilon}_{220} r_{22 i j}+\frac{1}{2}\left(\operatorname{Re} \frac{\partial v_{10}}{\partial x_{2}}+\frac{1}{\operatorname{Re}} \frac{\partial v_{20}}{\partial x_{1}}\right) r_{12 i j} .
\end{aligned}
$$

More details on the derivation of results in Eq. (11)-(16) and the meaning of relevant physical parameters can be referred in literatures [19, 24].

The criterion in Eq. (15) is a universal condition for evaluating the plastic flow stability of material in the plane strain state. The instability function $F_{\text {Inst }}$ represents the ratio of the thermal softening effect $R_{S} P_{0}$ to the strain hardening effect $F_{4} Q_{0}$ during the plastic deformation. In the numerator, the first term $F_{1} R_{S} P_{0}$ represents the softening effect of material produced by applied stress loading. The second term $F_{2} R_{0} R_{s} P_{0}$ represents the coupling effects of material softening and hardening originated from the strain rate sensitivity under complex stress loading, which is significant in the high-speed cutting. The third term $F_{3} R_{c} Q_{0} R_{s} P_{0}$ represents the coupling effects of the material softening and the strain hardening from different strain components, which is significant in the quasi-static loading case when the heat conduction effect becomes dominated. In the physical sense, if the value of instability function is larger than one, i.e., $F_{\text {Inst }}>1$, implying that the material softening effect overcomes the strain hardening effect, and the plastic flow of material is possibly unstable; whereas $F_{\text {Inst }} \leq 1$ indicates that the strain hardening effect of material is stronger than the material softening effect, and the plastic flow of material is certainly stable as a result. The expressions of the coefficient functions $F_{i}(i=1,2,3,4)$ indicate that this criterion can be readily generalized to the $3 \mathrm{D}$ loading situation. Moreover, since the values of functions $F_{2}$ and $F_{3}$ are constantly equal to zero under simple loading conditions, the coupling effects of material softening and hardening disappear naturally. Particularly, Eq. (15) degenerates into the criteria to determine the necking instability under the uniaxial tension loading and the shear banding instability under the simple shear loading if $F_{2}=F_{3}=0[34,35]$.
In this work, this criterion will be used to evaluate the stability of plastic flow of chip material in the 2D OCP, that is, if $F_{\text {Inst }}>1$, the chip flow is possibly unstable; otherwise, it is stable.

The derivation details of the criterion in Eq. (15) by the linear stability analysis can be found in references $[19,24]$. The condition $F_{\text {Inst }}>1$ results from the requirement that the characteristic in Eq. (11) has a real positive root, i.e., $\omega$ $>0$, leading to the increasing plastic strain with time as indicated by the inhomogeneous solution in Eq. (10). Notably, as one of the necessary conditions of plastic instability, $F_{\text {Inst }}>1$ simultaneously results in the unceasingly increasing flow stress. As a precursor of the final failure of materials, the occurrence of plastic instability implies that the material has lost bearing capacity. Therefore, the plastic instability certainly means that the true flow stress decreases and approaches zero as the loading continues. The criterion in Eq. (15) obtained from the linear stability analysis, however, does not consider the decreasing tendency of flow stress, and thus it only gives a necessary, not sufficient, condition for evaluating the plastic instability of materials. The necessary and sufficient condition for evaluating the plasticity stability should consider two aspects of factors. First, the value of instability function should be larger than one, and second, the true stressstrain relation has a negative hardening modulus during the plastic flow. That is, $F_{\text {Inst }}>1$ and $H=d \sigma / d \varepsilon<0$ must be met at the same time. In order to obtain the true stressstrain relation of chip material in the CFZ, we need to solve the initial-boundary value problem on the OCP. However, it is very difficult to obtain the exact solution due to the complex OCP even for a non-hardening plastic-rigid material [36]. Therefore, asymptotic fields involving stress, strain, and velocity of chip flow will be sought in terms of the 2D OCM. 


\subsection{Asymptotic fields}

To investigate the 2D plastic flow process of chip material, we found the asymptotic fields of the true stress, true strain, and velocity in the CFZ $O A B C$ (Fig. 5d). The boundary conditions regarding the velocity, velocity gradient, and stress are given as:

$$
\begin{aligned}
& O A:\left.v_{1}\right|_{O A}=\frac{\cos \alpha_{T}}{\cos \left(\varphi-\alpha_{T}\right)},\left.v_{2}\right|_{O A}=\sin \varphi,\left.\frac{\partial v_{2}}{\partial x_{1}}\right|_{O A}=\frac{\operatorname{Recos} \alpha_{T}}{\delta_{0} \cos \left(\varphi-\alpha_{T}\right)},\left.\frac{\partial v_{1}}{\partial x_{2}}\right|_{O A}=\frac{\cos \alpha_{T}}{\operatorname{Re} \delta_{0} \cos \left(\varphi-\alpha_{T}\right)} \\
& O C:\left\{\begin{array}{l}
\left.v_{1}\right|_{O C}=\sin \varphi \tan \left(\varphi-\alpha_{T}\right),\left.v_{2}\right|_{O C}=\sin \varphi,\left.\frac{\partial v_{1}}{\partial x_{1}}\right|_{O C}=-\left.\frac{\partial v_{2}}{\partial x_{2}}\right|_{O C}=\cos \varphi-\frac{\cos \alpha_{T}}{\cos \left(\varphi-\alpha_{T}\right)} \\
\left.\sigma_{N}\right|_{O C}=-\frac{\operatorname{Re} c_{1}}{2} \frac{\cos \alpha_{f}}{\cos \left(\varphi-\alpha_{T}\right)},\left.\sigma_{T}\right|_{O C}=\frac{\operatorname{Re} c_{1}}{2} \frac{\sin \alpha_{f}}{\cos \left(\varphi-\alpha_{T}\right)}
\end{array}\right. \\
& A B, B C:\left.\sigma_{N}\right|_{A B}=\left.\sigma_{T}\right|_{A B}=\left.\sigma_{N}\right|_{B C}=\left.\sigma_{T}\right|_{B C}=0
\end{aligned}
$$

where $c_{1}$ is the dimensionless wave velocity of work material. Equations (2)-(6) and boundary conditions in Eq. (17) constitute the complete formulation of the problem to be solved. By using the small parameter perturbation method, the linear dominating part of the inhomogeneous solution of the velocity field and the stress field are found as:

$$
\begin{aligned}
& v_{1}=\frac{\cos \varphi \cos \left(\varphi-\alpha_{T}\right)-\cos \alpha_{T}}{\cos \left(\varphi-\alpha_{T}\right)}\left(x_{1}-\frac{1}{2}\right)+\frac{\cos \alpha_{T}}{\cos \left(\varphi-\alpha_{T}\right)}\left(\frac{x_{2}}{\operatorname{Re} \delta_{0}}+1\right) \\
& v_{2}=\frac{\cos \alpha_{T}\left[2 \operatorname{Re} x_{1}-l_{0} \tan \left(\varphi-\alpha_{T}\right)\right]}{2 \delta_{0} \cos \left(\varphi-\alpha_{T}\right)}-\frac{\cos \varphi \cos \left(\varphi-\alpha_{T}\right)-\cos \alpha_{T}}{\cos \left(\varphi-\alpha_{T}\right)}\left(x_{2}-\frac{l_{0}}{2}\right)+\frac{\sin \varphi}{l_{0}},
\end{aligned}
$$

and

$$
\begin{aligned}
& \sigma_{12}=\operatorname{Re} I_{3}\left[I_{1} \operatorname{Re}\left(1-x_{1}\right)+I_{2}\left(l_{0}-x_{2}\right)\right] \\
& \sigma_{11}=\Sigma \cos \left(\varphi+\alpha_{f}-\alpha_{T}\right)+\sigma_{12} \tan \left(\varphi-\alpha_{T}\right) \\
& \sigma_{22}=\Sigma \sin \left(\varphi+\alpha_{f}-\alpha_{T}\right)+\sigma_{12} \tan \left(\varphi-\alpha_{T}\right) \\
& \Sigma=c_{1} I_{0} I_{3} \frac{\operatorname{Re}\left(x_{1}-1\right)-x_{2} \tan \left(\varphi-\alpha_{T}\right)}{2 \operatorname{Recos}^{2}\left(\varphi-\alpha_{T}\right)},
\end{aligned}
$$

where $\delta_{0}=0.17$ is the dimensionless PSZ width and $l_{0}=\sin (\varphi$ $\left.+\alpha_{T}-\alpha_{f}\right) / \cos \alpha_{f}$ contact length between the tool and chip normalized by PSZ length (Fig. $5 \mathrm{~d}$ ). The coefficients $I_{0}, I_{1}$, $I_{2}$, and $I_{3}$ in Eq. (19) have the forms:

$$
\begin{aligned}
& I_{0}=2 \cos \left(\varphi-\alpha_{T}\right) \cos \left(\alpha_{f}-\alpha_{T}\right)\left[1-\tan ^{2}\left(\varphi-\alpha_{T}\right)\right] \\
& I_{1}=\tan \left(\varphi-\alpha_{T}\right)(\cos \varphi-\sin \varphi) \\
& I_{2}=\cos \varphi-\sin \varphi \tan ^{2}\left(\varphi-\alpha_{T}\right) \\
& I_{3}=\left(\operatorname{Re}-\operatorname{Re}_{0}\right)^{2} \frac{1+B_{0} \gamma_{c}}{I_{1}+I_{2} l_{0}}
\end{aligned}
$$

where $\gamma_{c}$ is the shear failure strain of materials.

\section{Results and discussion}

As discussed above, the instability function $F_{\text {Inst }}$ in Eq. (15) involves a variety of material properties such as the thermal softening, strain hardening, strain rate sensitivity, heat conductivity and heat convection, the conversion between work and heat, and the applied loading conditions. Therefore, $F_{\text {Inst }}$ can be used to investigate the influence of material properties on the plastic flow stability during the cutting process. For the four testing metals, $F_{\text {Inst }}$ depends on Re differently because of the different thermophysical properties (Fig. 7). In the lowspeed cutting process (e.g., $\operatorname{Re}<2$ ), the material hardening effect $F_{2} R_{0}$ related to the strain rate sensitivity is not significant, whereas the strain hardening effect $F_{3} R_{c} Q_{0}$ caused by the heat conduction generates evident influence on the plastic deformation.

For Ti6Al4V alloy, the poor heat conductivity hinders the conduction of cutting heat which increases the temperature of chip materials. Consequently, the thermal softening effect influences the plastic deformation and thus $F_{\text {Inst }}$ always takes the positive values. Therefore, $0<F_{\text {Inst }} \leq 1$ indicates that the thermal softening takes effect, but is weaker than the strain hardening effect, and thus the plastic flow of chip material is stable.

For the other three metals, the good heat conductivity greatly promotes the heat conduction, resulting in the lower temperature rise and less thermal softening effect. The hardening effect related to the heat conductivity overweighing the material softening effect generated by the applied stress loadings can be reflected by the negative $F_{\text {Inst }}$. Physically, $F_{\text {Inst }}<0$ means that the plastic deformation is still in the strain hardening stage. The thermal softening phenomenon emerges only when the cutting speed is sufficiently large, i.e., $\operatorname{Re}>2$, and $F_{\text {Inst }}$ becomes positive in this situation. Therefore, $F_{\text {Inst }}<0$ means that the plastic deformation exhibits the pure strain hardening behavior, while $0<F_{\text {Inst }} \leq 1$ indicates that strain hardening and thermal softening come into play simultaneously.

The instability behaviors of plastic flow can be further evaluated by comparing $F_{\text {Inst }}$ with unity. For each testing metal, $\mathrm{Re}$ can be divided into three intervals: $\mathrm{Re}_{0}<\mathrm{Re}<\mathrm{Re}_{c 1}$, $\operatorname{Re}_{c 1}<\operatorname{Re}<\operatorname{Re}_{c}$ and $\operatorname{Re} \geq \operatorname{Re}_{c}$ (Fig. 7). For the lower range $\operatorname{Re}_{0}$ $<\operatorname{Re}<\operatorname{Re}_{c 1}, F_{\text {Inst }}<1$ is met, indicating that the plastic flow of 


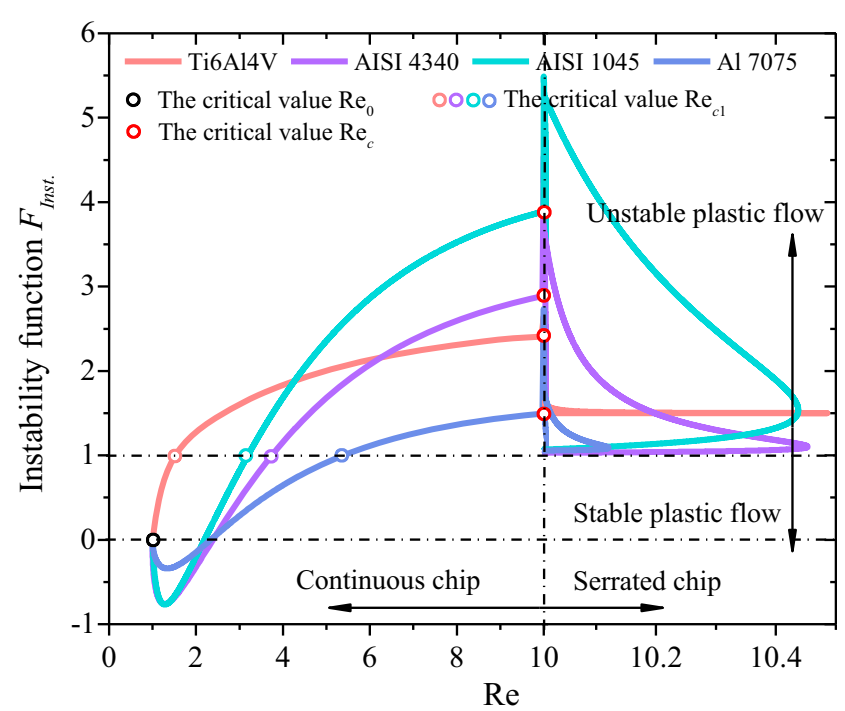

Fig. 7 The regulation of the instability function $F_{\text {Inst }}$ of four testing metals varying with $\mathrm{Re}$

continuous chip is stable. The parameter $\mathrm{Re}_{c 1}$ determines the upper limit of the steady plastic flow which can be called as the first critical parameter. The corresponding cutting speed is named as the first critical velocity denoted by $V_{c 1}$. For the higher range $\operatorname{Re} \geq \operatorname{Re}_{c}, F_{\text {Inst }}>1$ is met, indicative of the formation of serrated chips. The multiple shear banding instability results in the unsteady plastic flow of serrated chip [30]. The critical parameter $\operatorname{Re}_{c}=10$ corresponds to the transition of chip morphology from continuous to serrated. For the middle range $\mathrm{Re}_{c 1}<\operatorname{Re}<\operatorname{Re}_{c}, F_{\text {Inst }}>1$ is still met, implying that the plastic flow of continuous chip is possibly unstable. In order to finally determine the stability of plastic flow, it is necessary to further consider the true equivalent stress-strain relationship in the plastic flow process.

For the four testing metals, the true equivalent stresses of chip material with respect to Re are plotted in Fig. 8a. It can be seen that all metals have the maximum or minimum stress at the same $\mathrm{Re}$. $\mathrm{Re}=\mathrm{Re}_{c 2}=2.04$ with maximum stresses is defined as the second critical parameter, and the corresponding cutting speed $V_{c 2}$ is called as the second critical velocity. $\mathrm{Re}=\mathrm{Re}_{c}=10$ with the minimum stresses denotes the onset of the shear banding instability. On the other hand, Fig. 8b demonstrates that the true strain monotonically increases as $\mathrm{Re}$ increases. The maximum strain and the minimum stress at $\mathrm{Re}=\mathrm{Re}_{c}=10$ imply that the shear banding instability has occurred in PSZ which further leads to the formation of serrated chips. Combining with Fig. 8a, b, the equivalent stressstrain curves are plotted in Fig. 9 for all metals marked with critical Re. The stress increases as strain increases until Re $=$ $\mathrm{Re}_{c 2}=2.04$ for all metals, indicating that the plastic deformation is controlled by strain hardening and the plastic flow of continuous chip is stable. When $\operatorname{Re}_{c 2}<\operatorname{Re}<\operatorname{Re}_{c}$, the stress decreases as the strain increases. For the materials following the J-C constitutive model, the plastic flow stability depends on the competition between the thermal softening effect and the strain and strain rate hardening. When the true stress exceeds the strength limit, the flow stress decreases as the plastic strain increases, which indicates the hardening modulus become negative, i.e., $H=d \sigma / d \varepsilon<0$. This means that the plastic flow of chip material gets into the thermal softening stage. In this case, the necessary and sufficient condition of material plastic instability $F_{\text {Inst }}>1$ and $H<0$ are met simultaneously, and thus the plastic flow of continuous chip is unstable.

Figure 10 shows the dependence of the critical speeds $V_{c 1}$ and $V_{c 2}$ on Re. For clarity, these critical velocities are also listed in Table 3. It can be seen that when the cutting speed equals to these critical speeds, the onset of the plastic instability is possible since the condition $F_{\text {Inst }}>1$ is met. According to the necessary and sufficient conditions of plastic instability, the larger critical velocity should be the true critical speed for the plastic instability of continuous chip. Therefore, the critical speed of Ti6Al4V alloy is determined as $0.17 \mathrm{~m} / \mathrm{s}$ by
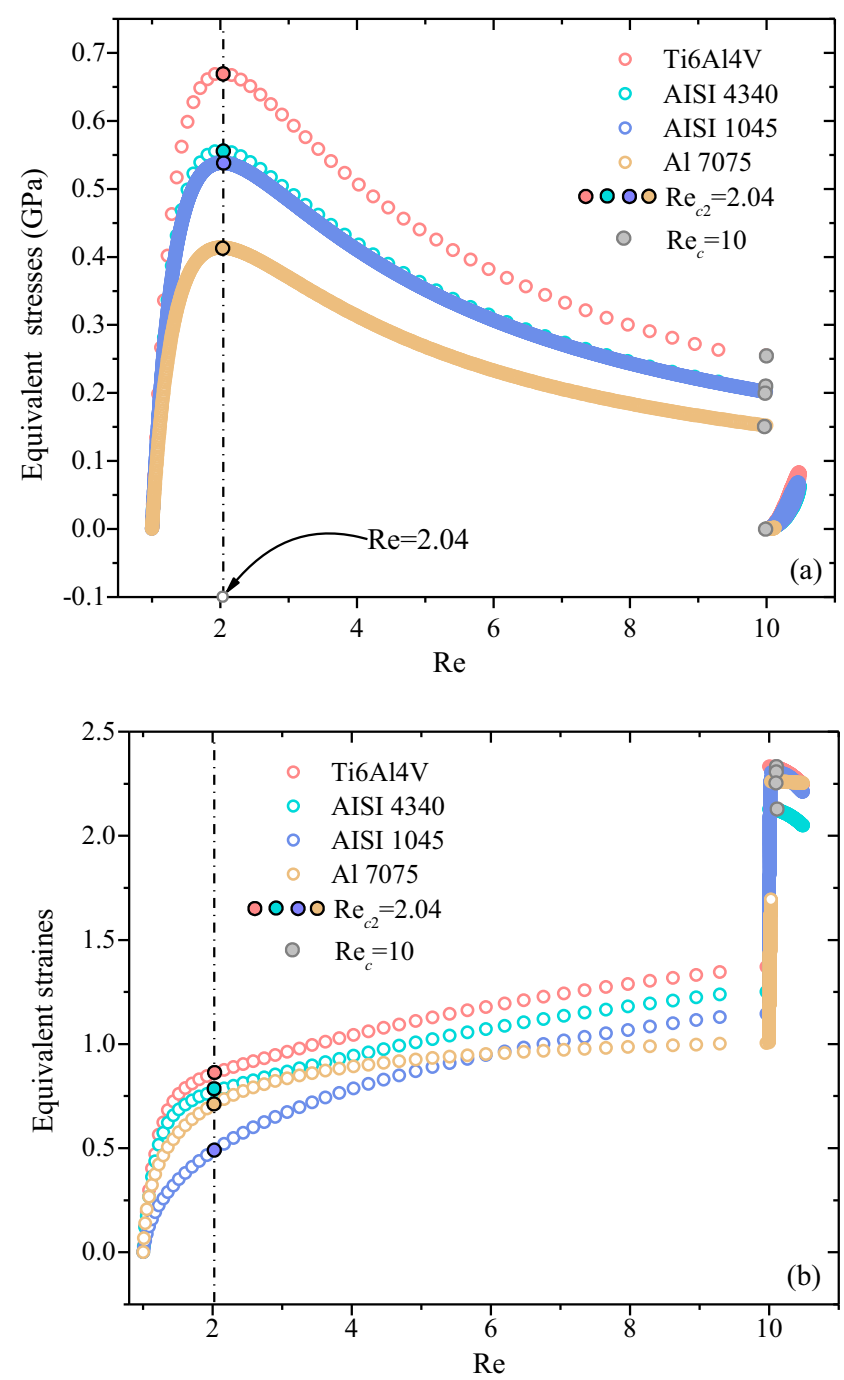

Fig. 8 The relationships of the equivalent stresses (a) and the equivalent strains (b) with the parameter Re for four testing metals 


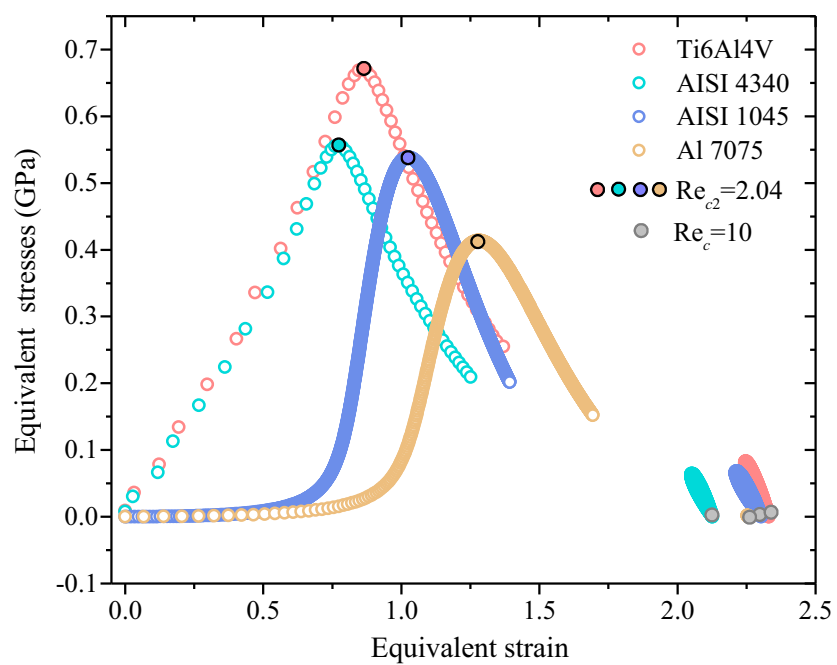

Fig. 9 The equivalent stress-strain relationship curves of four kinds of metals

conditional $\operatorname{Re}=\operatorname{Re}_{c 2}$. For the other three metals, the critical speeds are determined as Al 7075 alloy $\sim 3.6 \mathrm{~m} / \mathrm{s}$, AISI 4340 steel $\sim 19.24 \mathrm{~m} / \mathrm{s}$, and AISI 1045 steel $\sim 4.2 \mathrm{~m} / \mathrm{s}$, respectively. Nonetheless, there is always a critical speed in metal cutting process above which the plastic flow of continuous chip will become unstable.

It is worth emphasizing that the plastic instability of continuous chip is caused by 2D stress effects. Such special instability is characterized by non-localized deformation caused by the shear-compression composite stress. Obviously, it is different from the shear banding instability in the serrated chip produced by the simple shear stress in PSZ, which is characterized by shear localization deformation with thickness tens micrometers.

Theoretically, the two critical speeds of plastic instability in continuous chip should be strictly equal. Figure 10 shows that they are slightly different. One reason is that the velocity field

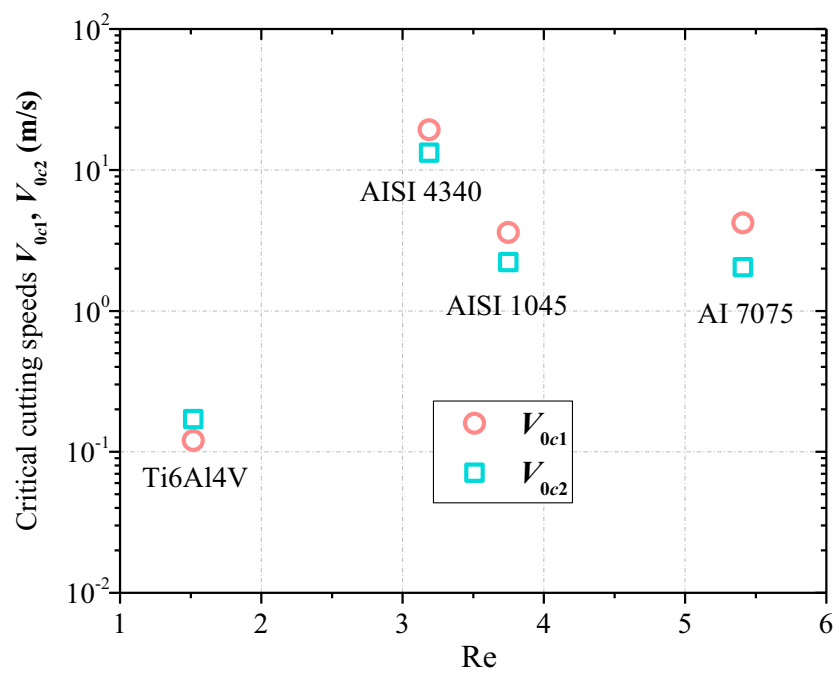

Fig. 10 The distribution of two kinds of critical cutting speeds and their comparison
Table 3 The critical parameters $\operatorname{Re}_{c 1}$ and $\mathrm{Re}_{c 2}$ and the corresponding critical cutting speeds $V_{0 c 1}$ and $V_{0 c 2}$

\begin{tabular}{lllll}
\hline & Ti6A14V & AISI 4340 & AISI 1045 & A1 7075 \\
\hline $\operatorname{Re}_{c 1}$ & 1.52 & 3.75 & 3.19 & 5.41 \\
$V_{c 1}(\mathrm{~m} / \mathrm{s})$ & 0.12 & 3.6 & 19.24 & 4.2 \\
$\operatorname{Re}_{c 2}$ & 2.04 & 2.04 & 2.04 & 2.04 \\
$V_{c 2}(\mathrm{~m} / \mathrm{s})$ & 0.17 & 2.21 & 13.26 & 2.04 \\
\hline
\end{tabular}

and stress field of plastic deformation of material in the CFZ $O A B C$ are the approximate solutions of the initial-boundary problem by using the small parameter perturbation method. Evidently, the field quantities in the CFZ should be non-linear functions of the Cartesian coordinates, while the approximate solutions Eqs. (18) and (19) are only the linear principal parts of the exact solution. In addition, for the sake of simplicity, the effects of temperature gradient, strain rate gradient, and mass convection are neglected in criterion Eq. (15), further leading to the difference of two critical speeds. Therefore, the two sets of critical speeds in Fig. 10 should be equal with each other for each testing material, which correspond to the identical critical parameter $\operatorname{Re}=\operatorname{Re}_{c 1}=\operatorname{Re}_{c 2}$. The modified Re describes the non-localized plastic instability of continuous chip during the cutting process. Besides, $\mathrm{Re}=\mathrm{Re}_{c}=10$ has been modified as the critical state that continuous chips transform into serrated ones by function $\chi\left(V_{0}\right)$, and thus the onset of shear banding instability in the PSZ can be determined by the same condition $\mathrm{Re}=\mathrm{Re}_{c}$. It can be seen that the parameter Re not only governs the plastic instability in continuous chips, but also shear banding instability in serrated chips. Therefore, Re is the dominating parameter that control the metal cutting process in a wide cutting speed. Moreover, Fig. 4 illustrates that both plastic instability in continuous chip and shear banding instability in serrated chips show the similar dependence on the thermophysical properties of work material (Fig. 4a) and the inertial effect produced by the high loading rate (Fig. 4b).

For the chip formation with different morphology, the dissipation mechanism of cutting energy is also different. The dissipation of cutting energy depends on the properties of work materials and the cutting conditions. Figure 11 shows the evolution of strain energy density with respect to equivalent strain. For a given cutting condition, the work done by the tool is transformed into two parts of plastic deformation energy: one part is taken away by moving chip material and the remaining part becomes the residual strain energy on machined surface. The less the residual strain energy is left on machined surface, the better machined surface quality we will have. Ti6Al4V alloy is generally considered as the hard-cut material because there is no significant strength decrease when the temperature is lower than the $\beta$-phase transition temperature about $1263 \mathrm{~K}$ [25]. Therefore, for Ti6Al4V alloy, more cutting energy is consumed to overcome larger cutting 
resistance than that of other three kinds of metals. Al 7075 alloy is easier to be machined because less energy is consumed in cutting process considering its sensitive temperature softening effect and lower strength. For the two mild steels, the consumed cutting energy depends on material properties of workpiece. AISI 4340 steel has higher initial yield strength and higher hardening modulus than that of AISI 1045 steel, and thus consumes more cutting energy under same cutting conditions.

The dissipation of cutting energy is related to the plastic flow stability of chip materials. Figure 11 shows that, for each testing metal, the dissipation of the cutting energy is affected by the chip morphology and formation mechanism in three ways. The continuous chips form in low-speed cutting process. The segment $O A$ represents the stable plastic flow and segment $A B$ denotes the unstable flow. The point $A$ on each curve is the inflection point when the stable plastic flow becomes unstable in continuous chip. In high-speed cutting process, segment $C$ denotes the unstable plastic flow of serrated chip. Physically, points $A, B$, and $C$ represent the maximum energy density dissipated by plastic deformation in the stable and unstable plastic flow processes of continuous chips and shear banding instability of serrated chips, respectively. Figure 12a shows that the mean ratio of the maximum energy densities between stable and unstable plastic flows in continuous chip is about 2.1. The energy density dissipated by the plastic flow inside shear bands is larger than that by the unstable plastic flow in continuous chip, and the mean ratio of maximum dissipative energy density to that of the stable flow of the continuous chip is about 2.7. Figure $12 \mathrm{~b}$ shows the total energy consumed by the plastic deformation in a sawtooth segment with the same size for serrated and continuous chips. The calculations show that, for the four testing metals, the cutting energy consumed by the unstable plastic flow of the

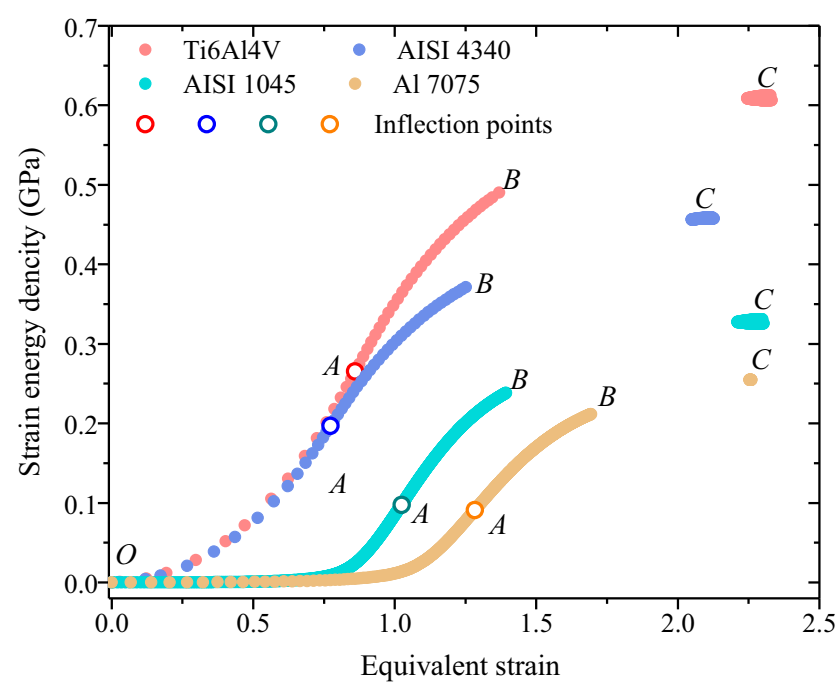

Fig. 11 The strain energy densities of plastic flow of chip material varying the equivalent plastic strain in the metal cutting
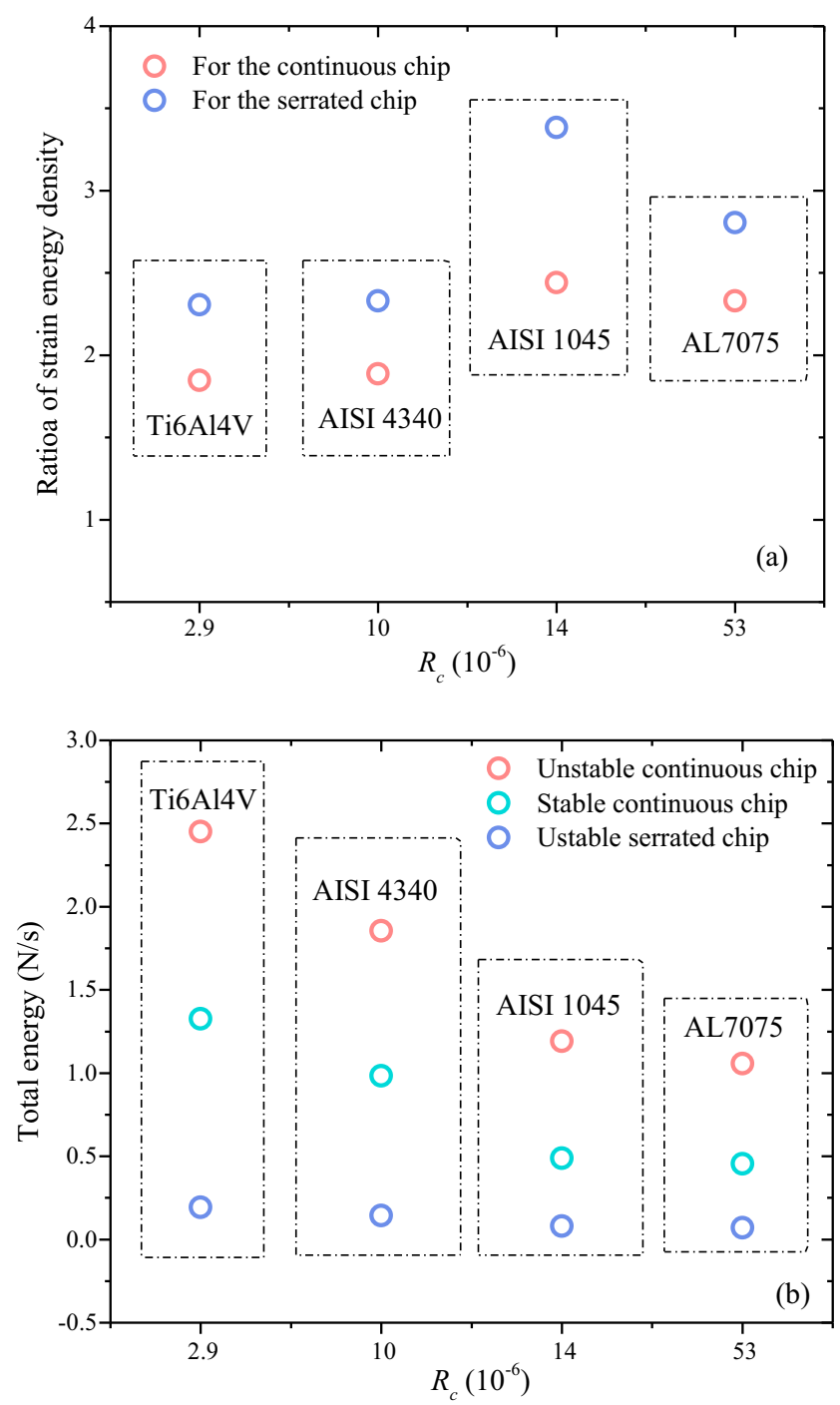

Fig. 12 The dependence of the ratios of the strain energy densities (a) and the total energy (b) on the parameter $R_{c}$ for the four testing metals

serrated chip is one order of magnitude lower than that consumed by the continuous chip. Therefore, when the serrated chips form in high-speed cutting process, less cutting energy transfers into the plastic deformation energy of chip material. Some of the remaining cutting energy is converted into the residual strain energy of machined surface, and the other is transformed into the kinetic energy of tool vibration. Tool vibration not only shortens the service life of tool, but also obviously affects the surface machining quality.

The dissipation of cutting energy strongly depends on the evolution of microstructure of chip materials during plastic flow. Figure 13 demonstrates the SEM observations of the microstructures of the as-received Ti6A14V alloy and of the longitudinal symmetric planes of the continuous and serrated chips obtained in OCP. Compared with the original microstructure of Ti6Al4V alloy (Fig. 13a), the original equiaxed grains are transformed into the slender grains with a length of 

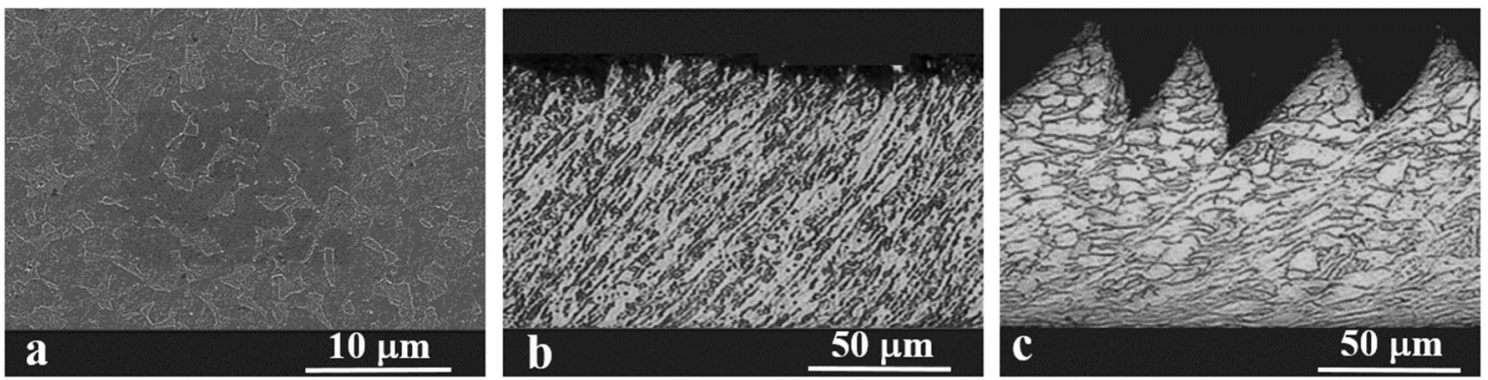

Fig. 13 Microstructure of a the as-received material; $\mathbf{b}, \mathbf{c}$ the chips obtained at a cutting speed of $0.05 \mathrm{~m} / \mathrm{s}$ and $0.8 \mathrm{~m} / \mathrm{s}$, respectively

about $30 \mu \mathrm{m}$ (Fig. 13b). Therefore, grains in continuous chip experiences severe but uniform shear deformation, which results in the uniform refinement of all grains. For the serrated chip forms in high-speed cutting process, however, the shear localization deformation leads to the severe refinement of grains only in the PSZ. The higher energy density dissipated by the plastic flow of PSZ indicates that the material inside the shear bands experienced the stronger shear deformation than the material in continuous chip. The grains outside shear bands are not obviously refined under the combined shearcompressive stress loading condition. The modelling results on the metal OCP show that the plastic strain outside PSZ is less than $0.2-0.3 \%$ [30] and thus, the material plastic deformation is still in the strengthening stage and not in unstable plastic flow.

From the viewpoint of engineering application, metal cutting should not only pursue the perfect machining quality, but also the higher production efficiency. The efficiency can be simply improved by increasing the cutting speed, but the formation of serrated chip leads to obvious deterioration of machining quality for most of metals. One can see that the formation of serrated chip requires less cutting energy, but the periodic shear banding instability transforms a considerable amount of the work done by the tool into the kinetic energy of tool vibration. This is the main factor that deteriorates the machined surface quality. In the low-speed cutting with continuous chip, the cutting energy consumed in the stable plastic flow is about half of the unstable plastic flow. Therefore, more cutting energy is transformed into the residual strain energy of machining surface, leading to the material softening by the dynamic recrystallization, the reversed strengthening, and the phase transformation. Moreover, it may also rough the machined surface and change the original mechanical properties of materials. The cutting thickness may become wavy on the next round cutting which aggravates the vibration during the cutting process [14], [38]. When the continuous chip flow is unstable, the tool vibration caused by the periodic shear banding instability does not present and more cutting energy is taken away by chip material as a result. The residual energy staying on machined surface is reduced significantly, and therefore, it might be the optimal instability mode for obtaining the better machined quality.
The experimental results in Fig. 3 demonstrate that as the cutting speed exceeds about $300 \mathrm{~m} / \mathrm{s}$, the serrated chips/debris tends to continuous again. One explanation believes that, in the ultra-high-speed cutting process, the large inertia effect produced by high-speed impact loadings suppresses the onset and evolution of shear bands in the serrated chip [29]. Another one is that, when the moving velocity of chip material along the rake surface is large enough to exceed the growth velocity of shear band along the shear plane, the shear bands would terminate inside the chip. The reason is that the materials under the shear localized deformation have moved out of the PSZ such that the shear stress loadings will not take effects on these materials before shear bands grow mature [9]. The rising question is if the ultra-high-speed cutting in which the continuous chip forms again can be a new machining technology for the better metal formability and workability. For now, the answer is still unknown. However, for the future development of metal machining, we have seen a good prospect from the current research.

\section{Conclusions}

In summary, we establish a 2D OCM and corresponding governing equations in this article to study the influence of 2D effects on plastic flow instability in the metal OCP. A controlling parameter $\mathrm{Re}$ is proposed to describe the plastic flow process and relevant stability behavior not just for the serrated chip, but also for the continuous chip. Furthermore, a universal criterion is established by linear stability analysis to evaluate the onset of plastic instability of chip materials. Combining with the true equivalent stress-strain relationship in plastic flow, the necessary and sufficient conditions $F_{\text {Inst }}>1$ and $H<0$ are proposed to evaluate of the plastic flow stability. Two instability modes of the plastic flow of chip materials are found as the shear localized instability causing the serrated chips and the plastic instability in the continuous chip owing to plane strain loading. The former is characterized by the strong localization of shear deformation, and the latter by severe non-localized shear deformation. Two critical speeds are related to these two instability modes which depend on the thermophysical properties of work material and the inertia 
effect of high rate loading. More importantly, our analysis regarding the energy dissipation mechanism indicates that the unstable plastic flow in the continuous chip may be the best instability mode for the better machining surface quality, vibration suppression, and the higher production efficiency.

Funding information This work was supported by the National Nature Science Foundation of China (Grant numbers: 11572337, 11772346, and 51575029).

\section{References}

1. Taylor FW (1906) On the art of cutting metals. Trans Am Soc Mech Eng XXVIII:31-350

2. Finnie I (1956) Review of the metal-cutting analysis of the past hundred years. Mech Eng 78:715-721

3. Shaw MC (1984) Metal cutting principles. Clarendon Press, Oxford

4. Arrazola PJ, Özel T, Umbrello D et al (2013) Recent advances in modelling of metal machining processes. CIRP Ann 62:695-718. https://doi.org/10.1016/J.CIRP.2013.05.006

5. Xu J, El Mansori M, Chen M, Ren F (2019) Orthogonal cutting mechanisms of CFRP/Ti6Al4V stacks. Int J Adv Manuf Technol. https://doi.org/10.1007/s00170-019-03734-x

6. Merchant ME (1945) Mechanics of the metal cutting process. I. Orthogonal cutting and a type 2 chip. J Appl Phys 16:267-275. https://doi.org/10.1063/1.1707586

7. Burns TJ, Davies MA (1997) Nonlinear Dynamics model for chip segmentation in machining. Phys Rev Lett 79:447-450. https://doi. org/10.1103/PhysRevLett.79.447

8. Burns TJ, Davies MA (2002) On repeated adiabatic shear band formation during high-speed machining. Int J Plast 18:487-506. https://doi.org/10.1016/S0749-6419(01)00006-7

9. Molinari A, Soldani X, Miguélez MH (2013) Adiabatic shear banding and scaling laws in chip formation with application to cutting of Ti-6Al-4V. J Mech Phys Solids 61:2331-2359. https:// doi.org/10.1016/J.JMPS.2013.05.006

10. Li Z, Guo H, Li L et al (2019) Study on surface quality and tool life in ultrasonic vibration countersinking of titanium alloys (Ti6A14V). Int J Adv Manuf Technol:1119-1137. https://doi.org/10.1007/ s00170-019-03572-x

11. Schneider F, Bischof R, Kirsch B et al (2016) Investigation of chip formation and surface integrity when micro-cutting cp-titanium with ultra-fine grain cemented carbide. Proc CIRP 45:115-118. https://doi.org/10.1016/J.PROCIR.2016.02.257

12. Atkins AG (2003) Modelling metal cutting using modern ductile fracture mechanics: quantitative explanations for some longstanding problems. Int J Mech Sci 45:373-396. https://doi. org/10.1016/S0020-7403(03)00040-7

13. Liyao G, Minjie W, Chunzheng D (2013) On adiabatic shear localized fracture during serrated chip evolution in high speed machining of hardened AISI 1045 steel. Int J Mech Sci 75:288-298. https://doi.org/10.1016/J.IJMECSCI.2013.07.004

14. Luo XK, Cheng K, Luo XC, Liu XW (2005) A simulated investigation on the machining instability and dynamic surface generation. Int J Adv Manuf Technol 26:718-725. https://doi.org/10.1007/ s00170-004-2057-z

15. Mahnama M, Movahhedy MR (2010) Prediction of machining chatter based on FEM simulation of chip formation under dynamic conditions. Int J Mach Tools Manuf 50:611-620. https://doi.org/10. 1016/J.IJMACHTOOLS.2010.03.009
16. Moufki A, Devillez A, Segreti M, Dudzinski D (2006) A semianalytical model of non-linear vibrations in orthogonal cutting and experimental validation. Int J Mach Tools Manuf 46:436449. https://doi.org/10.1016/J.IJMACHTOOLS.2005.04.017

17. Ye GG, Xue SF, Ma W et al (2012) Cutting AISI 1045 steel at very high speeds. Int J Mach Tools Manuf 56:1-9. https://doi.org/10. 1016/J.IJMACHTOOLS.2011.12.009

18. Wan L, Wang D, Gao Y (2016) The investigation of mechanism of serrated chip formation under different cutting speeds. Int J Adv Manuf Technol 82:951-959. https://doi.org/10.1007/s00170-0157409-3

19. Ma W, Chen X, Shuang F (2017) The chip-flow behaviors and formation mechanisms in the orthogonal cutting process of Ti6Al4V alloy. J Mech Phys Solids. https://doi.org/10.1016/j. jmps.2016.07.023

20. Wagner V, Baili M, Dessein G (2014) The relationship between the cutting speed, tool wear, and chip formation during Ti-5553 dry cutting. Int J Adv Manuf Technol 76:893-912. https://doi.org/10. 1007/s00170-014-6326-1

21. Piispanen V (1948) Theory of formation of metal chips. J Appl Phys 19:876-881. https://doi.org/10.1063/1.1697893

22. Palmer WB, Oxley PLB (1959) Mechanics of metal cutting. Proc Inst Mech Eng 173:623-645

23. Oxley PLB, Welsh MJM (1963) Calculating the shear angle in orthogonal metal cutting from fundamental stress, strain-rate properties of the work material. In: Proceedings 4th International Machine Tool Design and Research Conference. Pergamon, Oxford

24. Ma W, Li X, Dai L, Ling Z (2012) Instability criterion of materials in combined stress states and its application to orthogonal cutting process. Int J Plast 30-31:18-40. https://doi.org/10.1016/J.IJPLAS. 2011.09.003

25. Luo J, Li M, Li X, Shi Y (2010) Constitutive model for high temperature deformation of titanium alloys using internal state variables. Mech Mater 42:157-165. https://doi.org/10.1016/J. MECHMAT.2009.10.004

26. G.T. Gray III, S.R. Chen, W. Wright, M.F. Lopez (1994) Constitutive equations for annealed metals under compression at high strain rates and high temperatures. Los Alamos National Laboratory. LA-12669-MS. UC-000, Issued: January

27. Liu Y, Cai S, Shang X, Dai L (2017) Suppression of Hopf bifurcation in metal cutting by extrusion machining. Nonlinear Dyn 88: 433-453. https://doi.org/10.1007/s11071-016-3251-x

28. Johnson GR, Cook WH (1985) Fracture characteristics of three metals subjected to various strains, strain rates, temperatures and pressures. Eng Fract Mech 21:31-48. https://doi.org/10.1016/00137944(85)90052-9

29. Hortig C, Svendsen B (2007) Simulation of chip formation during high-speed cutting. J Mater Process Technol 186:66-76. https://doi. org/10.1016/J.JMATPROTEC.2006.12.018

30. Shuang F, Chen X, Ma W (2018) Numerical analysis of chip formation mechanisms in orthogonal cutting of Ti6Al4V alloy based on a CEL model. Int J Mater Form. https://doi.org/10.1007/s12289017-1341-z

31. Fang N (2003) Slip-line modeling of machining with a roundededge tool-Part I: new model and theory. J Mech Phys Solids 51: 715-742. https://doi.org/10.1016/S0022-5096(02)00060-1

32. Bisacre FFP, Bisacre GH (1947) The life of carbide-tipped turning tools. Proc Inst Mech Eng 157:452-469. https://doi.org/10.1243/ PIME_PROC_1947_157_070_02

33. Gioia $\overline{\mathrm{G}}$, Ortiz $\mathrm{M}(1 \overline{996})$ The two-dimensional structure of dynamic boundary layers and shear bands in thermoviscoplastic solids. J Mech Phys Solids 44:251-292. https://doi.org/10.1016/00225096(95)00071-2 
34. Bai YL (1982) Thermo-plastic instability in simple shear. J Mech Phys Solids 30:195-207. https://doi.org/10.1016/0022-5096(82) 90029-1

35. Molinari A (1997) Collective behavior and spacing of adiabatic shear bands. J Mech Phys Solids 45:1551-1575. https://doi.org/ 10.1016/S0022-5096(97)00012-4
36. Hill R (1950) The mathematical theory of plasticity. Oxford Univ. Press, Oxford

Publisher's note Springer Nature remains neutral with regard to jurisdictional claims in published maps and institutional affiliations. 